\title{
Trend-transformed independent vectors: Construction and stochastic comparison results
}

August 19, 2020

\author{
F. G. Badía ${ }^{* 1}$, Sophie Mercier $\dagger^{2}$ and C. Sangüesa $\ddagger 3$ \\ *Department of Statistical Methods and IUMA, University of Zaragoza, Zaragoza, \\ 50018, SPAIN \\ $\dagger$ Universite de Pau et des Pays de l'Adour, E2S UPPA, CNRS, LMAP, Pau, FRANCE \\ $\ddagger$ Department of Statistical Methods and IUMA, University of Zaragoza, Zaragoza, \\ 50009, SPAIN
}

\begin{abstract}
A new class of random vectors is introduced, where the components are obtained through trend-transforms of independent random variables. Such a vector is called trend-transformed independent vector. The new class includes many models from the previous literature, such as sequential and intermediate order statistics, or the vectors formed by the first arrival times in classical counting processes such as trend renewal/extended Pólya/non-homogeneous pure-birth processes. This allows to treat all these different models in a unified way. New multivariate stochastic comparison results are obtained between trend-transformed independent vectors with different parameters, which are shown to enlarge previous results from the literature.
\end{abstract}

2000 Mathematics subject classification: 62G30; 60E15; 60K10

Key words and phrases: Multivariate stochastic orders; Order statistics; Trend renewal process; Extended/Generalized Pólya process; Non-homogeneous pure-birth process.

\footnotetext{
${ }^{1}$ e-mail: gbadia@unizar.es

${ }^{2}$ sophie.mercier@univ-pau.fr

${ }^{3}$ Corresponding author e-mail: csangues@unizar.es
} 


\section{Introduction}

In [Lindqvist(2003)], B. Lindqvist \& al introduced a new type of counting process, which they called trend renewal processes. Such counting processes enlarge both renewal and non-homogeneous Poisson processes, which makes them quite flexible in an application context. For instance, in reliability theory, renewal and non-homogeneous Poisson processes are commonly used for the modeling of successive failures of a system subject to perfect or minimal instantaneous repairs, respectively. Trend renewal processes hence allow to model repairs whose efficiency can range from minimal up to perfect. Also, under technical assumptions, their arrival points exhibit interesting positive dependence properties as shown in [Badía et al(2018)b], where the authors also provide stochastic comparison results between trend renewal processes with different trend functions.

A trend renewal process is obtained through transforming the points of a renewal process by a so-called trend function $\Lambda$ (say). To be more specific, a vector $\left(T_{1}, \ldots, T_{n}\right)$ stands for the $n$ first points of a trend renewal process as soon as the increments (say) $X_{1}=\Lambda\left(T_{1}\right), X_{2}=\Lambda\left(T_{2}\right)-\Lambda\left(T_{1}\right), \ldots, X_{n}=$ $\Lambda\left(T_{n}\right)-\Lambda\left(T_{n-1}\right)$ are independent and identically distributed. We here suggest to enlarge this model in several ways:

1. The trend function $\Lambda$ can differ from one transformed inter-epoch time to the other, that is, in the definition of the increments (the $X_{i}$ 's), we set $X_{i}=\Lambda_{i}\left(T_{i}\right)-\Lambda_{i}\left(T_{i-1}\right)$, where the $\Lambda_{i}$ 's can differ from one $i$ to the other;

2. The $X_{i}$ 's are still required to be independent but not necessarily identically distributed;

3. The trend functions $\Lambda_{i}$ 's are not required to be strictly increasing any more nor assumed to be defined on $\mathbb{R}_{+}$(as for the original trend function from [Lindqvist(2003)]).

In this setting, the vector $\left(T_{1}, \ldots, T_{n}\right)$ is obtained through transforming the independent random variables $X_{1}, \ldots, X_{n}$ and we call it trend-transformed independent vector. Going back to the illustration in reliability theory, assumptions 1. and 2. allow to model some possible change of trend and/or distribution for the $X_{i}$ 's according to the number of failures already suffered by the system. Note that assumption 3. requires some care to deal with, wherever the inverse function of the trend function is required as we have to consider a generalized version of this inverse function. Also, the trend function can have a bounded support, which allows it to stand for the cumulative hazard rate of a bounded random variable, such as a beta or uniform one for instance.

All these extensions of the assumptions when compared with those for trendrenewal processes allow the class of trend-transformed independent vectors to encompass many other models from the literature and treat them in a unified way. For instance, the new class contains the vectors of arrival times in several classical processes in applied probability such as extended Pólya processes [Badía et al(2018a)] (which include the generalized Pólya processes from 
[Cha(2014)]) or general non-homogeneous pure-birth processes [Belzunce et al(2001)]. It also encompasses sequential order statistics [Zhuang et al(2007)] (which include the generalized order statistics from [Belzunce et al(2005)]) or intermediate order statistics [Badía et al(2018)b, Papadatos(1995)].

This paper first focuses on the construction of trend-transformed independent vectors under the general assumptions previously described, which is done in next section (Section 2), together with the derivation of first probabilistic properties and the development of several examples from the literature as specific cases. We next come to stochastic comparison results in Section 3, where trend-transformed independent vectors with different trend functions and/or different distributions for the increments (the $X_{i}$ 's) are considered. The results are provided in terms of the multivariate stochastic or likelihood ratio ordering. As we will see, they extend several other results from the literature, which were developed for specific trend-transformed independent vectors. Finally, concluding remarks and perspective are given in Section 4.

\section{Definition, construction and first properties}

We begin by defining what is called trend function in all the following.

Definition 1 A function $\Lambda(\cdot)$ is called a trend function if:

- There exists $M \in \mathbb{R} \cup\{\infty\}$ such that $\Lambda(\cdot):(-\infty, M) \rightarrow \mathbb{R}_{+}$,

- $\Lambda(\cdot)$ is non-decreasing and continuous on $(-\infty, M)$

- $\lim _{t \uparrow M} \Lambda(t)=\infty$.

- $\lim _{t \downarrow-\infty} \Lambda(t)=0$.

Remark 1 For some specific applications, $\Lambda(\cdot)$ will stand for the cumulative hazard rate function of a continuous random variable in the sequel. That is, if $X$ has a continuous distribution function $F$ with $F(x)<1, x<M$ and $\lim _{x \rightarrow M^{-}} F(x)=1$, then

$$
\Lambda(t)=-\ln (\bar{F}(t)), t \in(-\infty, M)
$$

is a trend function. When $M<\infty$, the restriction of the domain of $\Lambda(\cdot)$ to $(-\infty, M)$ allows $\Lambda(\cdot)$ to remain finite on its whole domain, even in case of a random variable with a bounded support.

Remark 2 The previous definition of trend function extends several similar notions from the literature. For instance, it enlarges the notion of cumulative intensity function used in [Badía et al(2018a)], where only non-negative random variables are considered, and therefore the domain of $\Lambda$ is of the shape $[0, M)$. It also enlarges the notion of trend function used in [Lindqvist(2003)] for the definition of trend-renewal processes (see Example 4 below), where the domain of $\Lambda$ is $\mathbb{R}_{+}$and where $\Lambda$ is assumed to be strictly increasing. As will be seen in 
the sequel, this last assumption can be avoided, but one needs to be very careful wherever the "inverse" of $\Lambda$ is required.

To better connect our model with others from the literature, we now come to the case where $\Lambda$ is absolutely continuous. We begin with defining so-called intensity functions.

Definition 2 A function $\lambda(\cdot)$ is called intensity function if:

- There exists $M \in \mathbb{R} \cup\{\infty\}$ such that $\lambda(\cdot):(-\infty, M) \rightarrow \mathbb{R}_{+}$,

- $\lambda(\cdot)$ is a measurable (Borel) function,

- $\int_{-\infty}^{t} \lambda(u) d u<+\infty$ for all $t<M$,

- $\int_{-\infty}^{M} \lambda(u) d u=\infty$.

Starting from an intensity function $\lambda(\cdot)$, it is easy to check that the function

$$
\Lambda(t):=\int_{-\infty}^{t} \lambda(u) d u,-\infty<t<M
$$

is a trend function. Conversely, starting from $\Lambda(\cdot)$, there exists $\lambda(\cdot)$ such that (2) is true as soon as $\Lambda(\cdot)$ is absolutely continuous.

We are now ready to define the notion of trend-transformed independent vector.

Definition 3 Let $\boldsymbol{\Lambda}=\left(\Lambda_{1}, \ldots, \Lambda_{n}\right)$ be a vector of trend functions such that $\Lambda_{i}$ is defined on $\left(-\infty, M_{i}\right), i=1,2, \ldots, n$ and $M_{i} \leq M_{i+1}, i=1,2, \ldots n-1$. Let $F_{1}, \ldots, F_{n}$ be continuous distribution functions on $\mathbb{R}_{+}$(so that $F_{i}(0)=0, i=$ $1, \ldots, n)$. Then, a random vector $\left(T_{1}, \ldots, T_{n}\right)$ is said to be a trend-transformed independent vector with trend vector $\boldsymbol{\Lambda}=\left(\Lambda_{1}, \ldots, \Lambda_{n}\right)$ and increment distribution vector $\mathbf{F}=\left(F_{1}, \ldots, F_{n}\right)$ (or parameter $(\boldsymbol{\Lambda}, \mathbf{F})$ for short) if

(a) $\mathbb{P}\left(T_{i}<M_{i}\right)=1, i=1,2, \ldots, n$;

(b) $\Lambda_{1}\left(T_{1}\right), \Lambda_{2}\left(T_{2}\right)-\Lambda_{2}\left(T_{1}\right), \ldots \Lambda_{n}\left(T_{n}\right)-\Lambda_{n}\left(T_{n-1}\right)$ are (non-negative) independent random variables, with respective distribution functions $F_{1}, \ldots, F_{n}$.

A trend-transformed independent sequence $\left(T_{n}\right)_{n=1,2, \ldots}$ with trend sequence $\left(\Lambda_{n}\right)_{n=1,2, \ldots}$, and increment distributions $\left(F_{n}\right)_{n=1,2, \ldots}$ is defined in a similar way, under similar assumptions for the $\Lambda_{n}$ 's and $F_{n}$ 's.

Remark 3 Assume $\left(T_{1}, \ldots, T_{n}\right)$ to be a trend-transformed independent vector with trend vector $\boldsymbol{\Lambda}=\left(\Lambda_{1}, \ldots, \Lambda_{n}\right)$ and increment distribution vector $\mathbf{F}=$ $\left(F_{1}, \ldots, F_{n}\right)$. Considering a vector $\left(a_{1}, \ldots, a_{n}\right)$ of (strictly) positive numbers, let us set $\hat{\Lambda}_{i}=a_{i} \Lambda_{i}$ and $\hat{F}_{i}(x)=F_{i}\left(x / a_{i}\right)$ for each $i \in\{1, \ldots, n\}$. Then it is easy to check that $\left(T_{1}, \ldots, T_{n}\right)$ still is a trend-transformed independent vector with trend vector $\hat{\boldsymbol{\Lambda}}=\left(\hat{\Lambda}_{1}, \ldots, \hat{\Lambda}_{n}\right)$ and increment distribution vector $\hat{\mathbf{F}}=\left(\hat{F}_{1}, \ldots, \hat{F}_{n}\right)$, 
whatever $a_{1}, \ldots, a_{n}$ are. This shows that different parameters $(\boldsymbol{\Lambda}, \mathbf{F})$ in Definition 3 can provide identical distributions for $\left(T_{1}, \ldots, T_{n}\right)$ and the model is not identifiable. A possibility might be to impose that the $F_{i}$ 's should have expected values equal to 1, as in [Lindqvist(2003)] for trend-renewal processes. We however prefer to keep the definition in its present form, which allows an easier way to identify models from the literature as specific trend-transformed independent vectors.

Starting from $(\boldsymbol{\Lambda}, \mathbf{F})$ as in the definition, we already know that each $\Lambda_{i}$ is continuous on $\left(-\infty, M_{i}\right), i=1, \ldots, n$. Assume further that each $\Lambda_{i}$ is increasing (and hence one-to-one) on $\left(-\infty, M_{i}\right)$. Then, it is easy to construct a trend-transformed independent vector with parameter $(\boldsymbol{\Lambda}, \mathbf{F})$ : Let us start from independent non negative random variables $X_{1}, \ldots, X_{n}$ such that $X_{i}$ has (continuous) distribution function $F_{i}, i=1, \ldots, n$ and let $T_{1}, \ldots, T_{n}$ be recursively defined through

$$
T_{1}=\Lambda_{1}^{-1}\left(X_{1}\right) \quad \text { and } \quad T_{i+1}=\Lambda_{i+1}^{-1}\left(X_{i+1}+\Lambda_{i+1}\left(T_{i}\right)\right), i=1, \ldots, n-1 .
$$

Note that based on $\mathbb{P}\left(X_{i}>0\right)=1, i=1, \ldots, n$, the random variables $T_{1}, \ldots, T_{n}$ are almost surely finite and hence well defined. Also, it is easy to check that $\left(T_{1}, \ldots, T_{n}\right)$ fulfills points (a) and (b) in the definition and it is a trend-transformed independent vector with parameter $(\boldsymbol{\Lambda}, \mathbf{F})$. Based on (3), it is clear that starting from independent random variables $X_{1}, \ldots, X_{n}$, the trend-transform allows to construct a random vector $\left(T_{1}, \ldots, T_{n}\right)$ where the inter-arrival times $\left(T_{1}, T_{2}-T_{1}, \ldots, T_{n}-T_{n-1}\right)$ are not independent any more. As will be seen in the following, a similar construction to (3) will remain valid even if the trend functions are not strictly increasing. More attention will be required however, for the handling of the inverse trend functions. As already told in the introduction, our model enlarges many others from the literature. We here provide first examples of well-known counting processes, for which successive arrival times are observed to follow a trend-transformed independent vector.

Example 4 (Trend renewal processes) Let $\Lambda$ be a trend function such that $\Lambda(0)=0$ and $M=\infty$ and let $F$ be a continuous distribution function on $\mathbb{R}_{+}$. Following the definition from [Lindqvist(2003)], the sequence of arrival times $\left(T_{n}\right)_{n=1,2, \ldots}$ in a trend renewal process with parameter $(\Lambda, F)$ can be seen as a trend-transformed independent sequence with parameters $\Lambda_{i}=\Lambda$ and $F_{i}=F$ for all $i \in \mathbb{N}^{*}$. Note that in [Berman (1981)], the authors consider a specific trend renewal process where $F$ is gamma distributed $\Gamma(\kappa, 1)$ and $\Lambda$ is as above. They observe that, when $\kappa \in \mathbb{N}^{*}$, then $\left(T_{1}, \ldots, T_{n}\right)$ is identically distributed as $\left(S_{\kappa}, S_{2 \kappa}, \ldots, S_{n \kappa}\right)$, where $\left(S_{n}\right)_{n=1,2, \ldots}$ is the sequence of arrival points in a non homogeneous Poisson process with cumulative intensity $\Lambda$. More generally, it is easy to check that, considering $\kappa_{1}, \ldots, \kappa_{n} \in \mathbb{N}^{*}$, then $\left(S_{\kappa_{1}}, S_{\kappa_{1}+\kappa_{2}}, \ldots, S_{\kappa_{1}+\ldots+\kappa_{n}}\right)$ is a trend-transformed independent vector, where $F_{i}$ is the gamma distribution function $\Gamma\left(\kappa_{i}, 1\right)$, for all $i=1, \ldots, n$.

Example 5 (Extended Pólya processes) Let $h(\cdot): \mathbb{N} \rightarrow \mathbb{R}_{+}^{*}$ and for each $i=1,2, \ldots$, let $F_{i}$ be the exponential distribution function with mean $1 / h(i-1)$. 
Also, let $\Lambda$ be a trend function on $[0, M)$. Then, based on [Badía et al(2018a), Prop. 2(b)], one can check that a trend-transformed independent sequence with parameters $\left(F_{i}, \Lambda_{i}=\Lambda\right), i=1,2, \ldots$ corresponds to the arrival times of an Extended Pólya process with parameter $(\Lambda, h)$. Based on Remark 3, a trendtransformed independent sequence with standard exponential increments (with mean 1) and $\Lambda_{i}=h(i-1) \Lambda$ correspond to the arrival times of the same Extended Pólya process with parameter $(\Lambda, h)$. As a by-product, the arrival times in a Generalized Pólya process as introduced by Cha [Cha(2014)] also form a trend-transformed independent sequence, as a Generalized Pólya process is a specific Extended Pólya process, where $h$ is a linear increasing function $(h(i)=\alpha i+\beta, i=1,2, \ldots, \alpha \geq 0, \beta>0)$.

We now come to the construction of a trend-transformed independent vector in the most general case, where the trend functions $\Lambda_{i}$ 's need not be (strictly) increasing. With that aim, let us consider a generic trend function $\Lambda$, which is non-decreasing and has range $\Lambda((-\infty, M))=\mathbb{R}_{+}$, whatever $M$ is. Then, we can introduce its generalized inverse function $\Lambda^{-1}: \mathbb{R}_{+} \rightarrow[-\infty, M)$, with:

$$
\Lambda^{-1}(s)=\inf \{t<M: \Lambda(t) \geq s\}=\sup \{t<M: \Lambda(t)<s\},
$$

for all $s>0$, and $\Lambda^{-1}(0)=-\infty$. The function $\Lambda^{-1}$ is known to be leftcontinuous on $(0, \infty)$ and such that $\Lambda\left(\Lambda^{-1}(s)\right)=s$ for all $s>0$ (based on the right-continuity of $\Lambda$ and on its range). Also, for all $s>0, t \in(-\infty, M)$, we have $\Lambda(t)<s$ if and only if $t<\Lambda^{-1}(s)$ (based on the right-continuity of $\Lambda$ ). Finally, $\Lambda^{-1}$ is (strictly) increasing, based on the continuity of $\Lambda$. See [Embrechts and Hofert(2013)] or [Boyer and Roux(2016)] for more details on generalized inverse functions. Note that for a given distribution function $G$, its quantile function $G^{-1}$ is usually defined as in (4), with $s \in[0,1]$.

If a trend function $\Lambda$ is not strictly increasing, we have to deal with the technical problem that its inverse function is not a continuous function. With that aim, let us introduce the set

$$
D:=\left\{s \in(-\infty, M) \mid \Lambda^{-1}(\Lambda(s))<s\right\} .
$$

In a similar way as in [Badía et al(2018a)], one can check that the set $D$ can be decomposed into (at most) countably many intervals, that is:

$$
D=\bigcup_{j \in J} I_{j}
$$

where $J$ is at most countable and $I_{j}=\left(a_{j}, b_{j}\right], j \in J$. Moreover $I_{j}$ represents an interval in which $\Lambda$ is a constant function. If $\Lambda(x)=0, x \leq m$, then the first interval is of the shape $(-\infty, m]$ and $\Lambda$ is strictly positive on the interval $(m, M)$. Note that if $\Lambda_{D^{c}}$ stands for the restriction of $\Lambda$ to $D^{c}$, the function $\Lambda_{D^{c}}$ now is a one-to-one function from $D^{c}$ to $\mathbb{R}_{+}$. As a consequence, for all $s>0$ and $t \in D^{c}$, we have $\Lambda(t)>s$ if and only if $t>\Lambda_{D^{c}}^{-1}(s)$, namely if and only $t>\Lambda^{-1}(s)$. 
For each $\Lambda_{i}$ in Definition 3, the corresponding set is denoted by $D_{i}$. Next Lemma shows that in a trend-transformed independent vector $\left(T_{1}, \ldots, T_{n}\right)$, the probability that $T_{i}$ belongs to the set $D_{i}$ is zero, for each $i=1, \ldots, n$.

Lemma 6 Let $\boldsymbol{\Lambda}=\left(\Lambda_{1}, \ldots, \Lambda_{n}\right)$ be a vector of trend functions and let $\mathbf{F}=$ $\left(F_{1}, \ldots, F_{n}\right)$ be an increment distribution vector, as in Definition 3. Let $\left(T_{1}, \ldots, T_{n}\right)$ be a trend-transformed independent vector with parameter $(\boldsymbol{\Lambda}, \mathbf{F})$. Let $X_{1}=$ $\Lambda_{1}\left(T_{1}\right)$ and $X_{i}=\Lambda_{i}\left(T_{i}\right)-\Lambda_{i}\left(T_{i-1}\right), i=2, \ldots, n$. Then we have

$$
\mathbb{P}\left(T_{i} \in D_{i}^{c} \text { for all } i=1, \ldots, n\right)=1,
$$

where the $D_{i}$ 's are the sets defined in (5). Also, (3) holds with probability 1.

Proof. Assume first that Equation (7) is true. Then we have

$$
\begin{aligned}
1=\mathbb{P}\left(\Lambda_{i}\left(T_{i}\right)=X_{i}+\Lambda_{i}\left(T_{i-1}\right)\right) & =\mathbb{P}\left(\Lambda_{i}\left(T_{i}\right)=X_{i}+\Lambda_{i}\left(T_{i-1}\right), T_{i} \in D_{i}^{c}\right) \\
& =\mathbb{P}\left(T_{i}=\Lambda_{i}^{-1}\left(X_{i}+\Lambda_{i}\left(T_{i-1}\right)\right)\right)
\end{aligned}
$$

for each $i=2, \ldots, n$, as $\Lambda_{i}^{-1}\left(\Lambda_{i}(s)\right)=s$ for all $s \in D_{i}^{c}$. Also, $\mathbb{P}\left(T_{1}=\Lambda_{1}^{-1}\left(X_{1}\right)\right)=$ 1 in a similar way. Thus, Equation (7) implies that (3) is verified for all $i=1,2, \ldots, n$ with probability 1 . Hence the only point to prove is Equation (7).

Now Equation (7) can be readily seen to be equivalent to

$$
\mathbb{P}\left(T_{i} \in D_{i}\right)=0, \quad \text { for all } i=1,2, \ldots, n,
$$

which we next show by induction. For the case $i=1$, let us consider any interval $I_{1 j}=\left(a_{1 j}, b_{1 j}\right] \subseteq D_{1}$. As $\Lambda_{1}$ is constant on $I_{1 j}$, we have

$$
\mathbb{P}\left(T_{1} \in I_{1 j}\right)=\mathbb{P}\left(\Lambda_{1}\left(T_{1}\right)=\Lambda_{1}\left(b_{1 j}\right)\right)=0,
$$

because $X_{1}=\Lambda_{1}\left(T_{1}\right)$ has a continuous distribution function for the last equality. Hence $\mathbb{P}\left(T_{1} \in D_{1}\right)=0$. Similarly, let us take $1<k \leq n$ and assume that $\mathbb{P}\left(T_{i} \in D_{i}\right)=0$ holds true for each $i=1, \ldots, k-1$. To show the result for $k$, let us notice that from the first part of this proof, we know that $T_{i}=\Lambda_{i}^{-1}\left(X_{i}+\right.$ $\left.\Lambda_{i}\left(T_{i-1}\right)\right)$ holds almost surely for each $i=2, \ldots, k-1$. Hence $T_{k-1}$ depends only on $X_{1}, \ldots, X_{k-1}$ (almost surely) and the random variables $X_{k}$ and $T_{k-1}$ are independent. Based on

$$
\Lambda_{k}\left(T_{k}\right)=X_{k}+\Lambda_{k}\left(T_{k-1}\right),
$$

we derive that $\Lambda_{k}\left(T_{k}\right)$ is a convolution between $T_{k-1}$ and the continuous random variable $X_{k}$. Hence $\Lambda_{k}\left(T_{k}\right)$ also is a continuous random variable. Thus, as in the first case, let us consider any interval $I_{k j}=\left(a_{k j}, b_{k j}\right] \subseteq D_{k}$. We have

$$
\mathbb{P}\left(T_{k} \in I_{k j}\right)=\mathbb{P}\left(\Lambda_{k}\left(T_{k}\right)=\Lambda_{k}\left(b_{k j}\right)\right)=0,
$$

and therefore $\mathbb{P}\left(T_{k} \in D_{k}\right)=0$. Hence (8) has been verified, which achieves the proof.

We have the following result, which shows us that a vector $\left(T_{1}, \ldots, T_{n}\right)$ as in Definition 3 is unequivocally determined using (3). 
Proposition $\mathbf{7}$ Let $\boldsymbol{\Lambda}=\left(\Lambda_{1}, \ldots, \Lambda_{n}\right)$ be a vector of trend functions and let $\mathbf{F}=\left(F_{1}, \ldots, F_{n}\right)$ be an increment distribution vector, as in Definition 3.

(a) Let $X_{1}, \ldots, X_{n}$ be independent random variables, where each $X_{i}$ has distribution function $F_{i}, i=1, \ldots, n$. Then the random vector $\left(T_{1}, \ldots, T_{n}\right)$ constructed by (3) is a trend-transformed independent vector with parame$\operatorname{ter}(\boldsymbol{\Lambda}, \mathbf{F})$.

(b) Let $\left(T_{1}, \ldots, T_{n}\right)$ be a trend-transformed independent vector with parameter $(\boldsymbol{\Lambda}, \mathbf{F})$ and let us set $X_{1}=\Lambda_{1}\left(T_{1}\right)$ and $X_{i}=\Lambda_{i}\left(T_{i}\right)-\Lambda_{i}\left(T_{i-1}\right), i=2, \ldots, n$. Then, (3) is satisfied with probability 1.

Proof. We only show point (a), as point (b) has already been proved in Lemma 6 . Then, let $\left(T_{1}, \ldots, T_{n}\right)$ be constructed by (3), as described in point (a). Let us first show that this construction provides a random vector with a proper distribution, that is that there is no "escape" of mass to $-\infty$. More specifically, let us show that

$$
\mathbb{P}\left(T_{i}>-\infty\right)=1, \quad i=1,2, \ldots, n .
$$

To show that, let us first note that for any trend function $\Lambda$, we have

$$
\Lambda^{-1}(y)>-\infty \text { if and only if } y>0 .
$$

Indeed, if $y>0$, then there exists $t<M$ such that $\Lambda(t)<y$. Hence

$$
\Lambda^{-1}(y)=\sup \{t<M: \Lambda(t)<y\}>-\infty .
$$

Based on $\Lambda^{-1}(0)=-\infty$, the converse is clear and (10) is true. Thus, by the previous property and (3):

$$
\mathbb{P}\left(T_{1}>-\infty\right)=\mathbb{P}\left(X_{1}>0\right)=1,
$$

as $F_{1}(0)=0$. The other cases $i=2,3, \ldots, n$ in (9) are proved in a similar way. Then it remains to show that $\left(T_{1}, \ldots, T_{n}\right)$ fulfills conditions (a) and (b) in Definition 3. Condition (a) is clear. As for condition (b), note that (3) and the property $\Lambda\left(\Lambda^{-1}(s)\right)=s, s>0$ allow us to write

$$
\Lambda_{1}\left(T_{1}\right)=X_{1} \quad \text { and } \quad \Lambda_{i+1}\left(T_{i+1}\right)=X_{i+1}+\Lambda_{i+1}\left(T_{i}\right) .
$$

Thus, condition (b) from Definition 3 is also satisfied, which achieves the proof.

Remark 4 (a) Though it is not assumed in Definition 3, we always have $T_{i} \leq$ $T_{i+1}, i=1,2, \ldots, n-1$ almost surely (a.s.), for any trend-transformed independent vector $\left(T_{1}, \ldots, T_{n}\right)$. Indeed, based on (3), we know that $T_{i+1} \geq$ $\Lambda_{i+1}^{-1}\left(\Lambda_{i+1}\left(T_{i}\right)\right) \quad$ a.s. If $T_{i} \in D_{i+1}^{c}$, then $\Lambda_{i+1}^{-1}\left(\Lambda_{i+1}\left(T_{i}\right)\right)=T_{i}$ a.s. and the result is clear. If $T_{i} \in\left(a_{i, j+1}, b_{i, j+1}\right] \subseteq D_{i+1}$, then $b_{i, j+1}<T_{i+1}$ because $T_{i+1} \in D_{i+1}^{c}$ and

$$
\Lambda_{i+1}\left(T_{i+1}\right) \geq \Lambda_{i+1}\left(T_{i}\right)=\Lambda_{i+1}\left(b_{i, j+1}\right) .
$$

This entails that $T_{i} \leq b_{i, j+1}<T_{i+1}$ a.s. and the required result. 
(b) In the specific case where $\Lambda_{i}=\Lambda, i=1, \ldots, n$, it is easy to check that

$$
\Lambda\left(T_{i}\right)=X_{1}+\ldots+X_{i}, \quad i=1, \ldots, n,
$$

which entails that

$$
T_{i}=\Lambda^{-1}\left(X_{1}+\ldots+X_{i}\right), \quad i=1, \ldots, n
$$

because $T_{i} \in D^{c}$.

(c) Assume that $\left(\Lambda_{1}, \ldots, \Lambda_{n}\right)$ are the cumulative hazard rates of some distribution functions $\left(G_{1}, \ldots, G_{n}\right)$, as in (1). Note that $\Lambda_{i}=L \circ G_{i}$, where $L(x)=-\ln (1-x), \quad 0 \leq x<1$. Thus, using [Embrechts and Hofert(2013), Prop 1(8)], $\Lambda_{i}^{-1}=G_{i}^{-1} \circ L^{-1}$. As $L^{-1}(u)=1-e^{-u}, \quad u \geq 0$, we can write

$$
\Lambda_{i}^{-1}(u)=G_{i}^{-1}\left(1-e^{-u}\right), \quad u \geq 0,
$$

and therefore, (3) can be alternatively expressed in terms of the quantile functions $\left(G_{1}^{-1}, \ldots, G_{n}^{-1}\right)$.

Now we are in a position to give the following additional examples included in our model.

Example 8 (Sequential order statistics and generalized order statistics) Sequential order statistics (SOS) include many examples of ordered random variables. They have been intensively studied from their introduction in Kamps [Kamps(1995b)] to nowadays. In particular, Cramer and Kamps [Cramer and Kamps (2003)] gave an alternative definition of SOS which is coincident with the one in [Kamps(1995b)] for continuous random variables, and inspired the following definition given in [Lenz(2008), p.37] (see also [Torrado(2012)]). For given distribution functions $G_{1}, \ldots, G_{n}$ with $G_{1}^{-1}(1) \leq \ldots \leq G_{n}^{-1}(1)$, their sequential order statistics are recursively defined as $X_{0, n}^{\star}=-\infty$ and

$$
X_{i, n}^{\star}=G_{i}^{-1}\left(1-U_{i} \bar{G}_{i}\left(X_{i-1, n}^{\star}\right)\right),
$$

where $\left(U_{1}, \ldots, U_{n}\right)$ is a vector of independent uniform random variables on the interval $(0,1)$. This is a slightly different definition than the one used in [Kamps(1995b), Cramer and Kamps (2003)]. The original definition of SOS (with associate distributions $G_{i}^{\star}$ ) is obtained by (14), by means of the substitution

$$
G_{i}^{\star}(t)=1-\left(1-G_{i}(t)\right)^{1 /(n-i+1)} .
$$

In order to see that sequential order statistics satisfy (3) and, therefore, Definition 3, let us consider $M_{i}=G_{i}^{-1}(1), i=1,2 \ldots, n$ and let $\boldsymbol{\Lambda}=\left(\Lambda_{1}, \ldots, \Lambda_{n}\right)$ be the vector of cumulative hazard rate functions associated to $\left(G_{1}, \ldots, G_{n}\right)$, as defined in (1) Now consider $\left(X_{1}, \ldots, X_{n}\right)$ the random vector with $X_{i}=-\ln \left(U_{i}\right)$, $i=1, \ldots, n$. It is readily seen that $X_{i}$ are exponential random variables with mean 1. Now consider the vector $\left(T_{1}, \ldots, T_{n}\right)$ as defined in (3). Using (13) this expression can be rewritten as

$$
T_{i}=\Lambda_{i}^{-1}\left(X_{i}+\Lambda_{i}\left(T_{i-1}\right)\right)=G_{i}^{-1}\left(1-e^{-X_{i}} e^{-\Lambda_{i}\left(T_{i-1}\right)}\right)=G_{i}^{-1}\left(1-U_{i} \bar{G}_{i}\left(T_{i-1}\right)\right),
$$


which coincides with (14), thus obtaining that $\left(T_{1}, \ldots, T_{n}\right)={ }_{s t}\left(X_{1, n}^{\star}, \ldots, X_{1, n}^{\star}\right)$. In other words, sequential order statistics are trend-transformed independent vectors with trend vector defined as the cumulative hazard rates of $G_{1}, \ldots, G_{n}$ and exponential increment distributions with rate 1. Finally, remember that generalized order statistics (GOS) are a particular case of sequential order statistics, where $G_{i}=G^{a_{i}}, i=1, \ldots, n$. In that case, the cumulative hazard rates of each $G_{i}$ is $\Lambda_{i}=a_{i} \Lambda$, where $\Lambda$ is the cumulative hazard rate of $G$. Hence, GOS are trend-transformed independent vectors with $\Lambda_{i}=a_{i} \Lambda$ and exponential increment distributions with rate 1. Equivalently (recall Remark 3), they can be seen as trend-transformed independent vectors with $\Lambda_{i}=\Lambda$ and exponential increment distributions with mean $1 / a_{i}$. Note that they can also be seen as Generalized Pólya processes (see Example 5), as already noticed in [Badía et al(2018a)].

Example 9 (Non-homogeneous pure-birth processes) Following the study by Belzunce et al. [Belzunce et al(2001)], a non-homogeneous pure-birth process is a counting processes $(N(t), t \geq 0)$ provided with a Markovian structure, in which for each $i=0,1, \ldots, \lambda_{i}(t)$ is an intensity function on $\mathbb{R}_{+}$such that

$$
\lim _{h \downarrow 0} \mathbb{P}(N(t+h)-N(t)=1 \mid N(t)=i)=\lambda_{i}(t), \quad t \geq 0
$$

As noticed by [Lenz(2008), p.39], the $n$ first interarrival times of this class of processes are sequential order statistics in which the associated distribution functions are those with cumulative hazard rates

$$
\Lambda_{i}(t)=\int_{0}^{t} \lambda_{i}(u) d u, \quad t \geq 0 \quad(0 \text { if } t<0) .
$$

Thus, the arrival times in a non-homogeneous pure-birth processes are a particular case of trend-transformed i.i.d. exponential random variables with mean 1.

Example 10 (Intermediate order statistics) The class of intermediate order statistics was considered in the pioneer paper by Papadatos [Papadatos(1995)]. Their definition is based on the ordered Dirichlet distribution defined as follows. $A$ random vector $\mathbf{U}=\left(U_{1}, \ldots, U_{n}\right)$ follows the ordered Dirichlet distribution with parameters $\nu_{i}>0, i=1,2, \ldots, n+1$, if its joint $p d f$ is given by

$$
\frac{\Gamma\left(\nu_{1}+\nu_{2}+\cdots+\nu_{n+1}\right)}{\Gamma\left(\nu_{1}\right) \Gamma\left(\nu_{2}\right) \cdots \Gamma\left(\nu_{n+1}\right)} x_{1}^{\nu_{1}-1} \prod_{i=2}^{n}\left(x_{i}-x_{i-1}\right)^{\nu_{i}-1}\left(1-x_{n}\right)^{\nu_{n+1}-1},
$$

for $0 \leq x_{1} \leq x_{2} \leq \cdots \leq x_{n} \leq 1$ (0 otherwise), where $\Gamma$ is the standard gamma function. Let $0=p_{0}<1 \leq p_{1}<p_{2}<\cdots<p_{n} \leq n$. The intermediate order statistics of order $p_{1}, \ldots, p_{n}$ issued from a distribution function $G$ is the random vector $\left(W_{1}:=G^{-1}\left(U_{1}\right), \ldots, W_{n}:=G^{-1}\left(U_{n}\right)\right)$, where $\left(U_{1}, \ldots, U_{n}\right)$ is an ordered Dirichlet random vector with parameters $\nu_{i}=p_{i}-p_{i-1}, i=1,2, \ldots, n$, and $\nu_{n+1}=n+1-p_{n}$. In the case where $p_{i}=i, i=1,2, \ldots, n$ these intermediate order statistics are the classical order statistics. As noticed by 
[Beutner and Kamps(2008)], the baseline random vector $\left(U_{1}, \ldots, U_{n}\right)$ can be written with respect to independent beta random variables as follows:

$$
U_{i}=1-\prod_{j=1}^{i} B_{j}, \quad i=1,2, \ldots, n
$$

where $B_{j}$ is a beta random variable with parameters $a=n-p_{j}+1$ and $b=$

$p_{j}-p_{j-1}$. Let us recall that the beta distribution with parameters $a>0$ and $b>0$ admits the following $p d f$ :

$$
\frac{1}{B(a, b)} x^{a-1}(1-x)^{b-1}, \quad 0 \leq x \leq 1
$$

where $B(.,$.$) is the standard beta function. Setting \Lambda$ to be the cumulative hazard rate associated to $G$ and using (13), we now get

$$
W_{i}=G^{-1}\left(1-\prod_{j=1}^{i} B_{j}\right)=G^{-1}\left(1-e^{\ln \left(\prod_{j=1}^{i} B_{j}\right)}\right)=\Lambda^{-1}\left(-\sum_{j=1}^{i} \ln \left(B_{j}\right)\right)
$$

and therefore, using Remark 4(b), intermediate order statistics are trend-transformed independent vectors with trend vector $\left(\Lambda_{1}=\Lambda, \ldots, \Lambda_{n}=\Lambda\right)$ and increments defined as $X_{i}=-\ln \left(B_{i}\right), i=1, \ldots, n$. Note that if $B$ is a beta distributed random variable with parameters $a>0$ and $b>0$, then the density of $X=-\ln (B)$ is given by

$$
\frac{1}{B(a, b)} e^{-a y}\left(1-e^{-y}\right)^{b-1}, \quad 0 \leq y<\infty
$$

\section{Multivariate dependence properties and stochas- tic comparisons results}

Our aim in this section is to obtain multivariate dependence properties between the components of a trend-transformed independent vector, as well as stochastic comparison results. Those results are of practical interest, for instance, in reliability theory: If we consider that $\left(T_{1}, \ldots, T_{n}\right)$ represent successive failure times of a device subject to instantaneous (imperfect) repairs at failure, stochastic comparison results between two different trend-transformed independent vectors allow to compare the failure times of two different devices and know which one is expected to have shorter failure times for instance. In the same reliability context, a positive dependence property between successive failure times means that the later the arrivals of the first failures, the later the following failures are expected to occur. Negative dependence properties mean the contrary. These concepts are dealt with in many text books of applied probability, see for instance [Müller and Stoyan(2002)]. 
There are also several monographic books dealing with stochastic orders, see for instance [Shaked and Shanthikumar(2007)] .

In some places and under appropriate conditions, we shall need the joint density function of $\left(T_{1}, \ldots, T_{n}\right)$, that we now provide.

Proposition 11 Let $\left(T_{1}, \ldots, T_{n}\right)$ be a trend-transformed independent vector with trend function $\boldsymbol{\Lambda}=\left(\Lambda_{1}, \ldots, \Lambda_{n}\right)$ and increment distributions $\mathbf{F}=\left(F_{1}, \ldots, F_{n}\right)$. If each $\Lambda_{i}$ is absolutely continuous with corresponding intensity function $\lambda_{i}$ and if each $F_{i}$ admits a density $f_{i}$, then the joint density function for $\left(T_{1}, \ldots, T_{n}\right)$ exists and is given by

$$
f_{\left(T_{1}, \ldots, T_{n}\right)}\left(t_{1}, \ldots, t_{n}\right)=\prod_{i=1}^{n} \lambda_{i}\left(t_{i}\right) \prod_{i=1}^{n} f_{i}\left(\Lambda_{i}\left(t_{i}\right)-\Lambda_{i}\left(t_{i-1}\right)\right) 1_{A}\left(t_{1}, \ldots, t_{n}\right)
$$

(0 otherwise), where $A:=\left\{\left(t_{1}, \ldots, t_{n}\right) \in \mathbb{R}^{n} \mid-\infty=t_{0}<t_{1}<t_{2}<\cdots<\right.$ $\left.t_{n}, t_{i}<M_{i}, i=1, \ldots, n\right\}$.

Proof. The existence of the joint density can easily be proved recursively, starting from the construction of $\left(T_{1}, \ldots, T_{n}\right)$ given in (3) and we skip the details. Now, using successive conditioning and based on the fact that $T_{i+1}$ depends on $\left(T_{1}, \ldots, T_{i}\right)$ only through $T_{i}$, we may write

$$
f_{\left(T_{1}, \ldots, T_{n}\right)}\left(t_{1}, \ldots, t_{n}\right)=f_{T_{1}}\left(t_{1}\right) \prod_{i=1}^{n-1} f_{T_{i+1} \mid T_{i}=t_{i}}\left(t_{i+1}\right) .
$$

Based on (3) and on the independence between $X_{i+1}$ and $T_{i}$, we have

$$
\begin{aligned}
f_{T_{i+1} \mid T_{i}=t_{i}}\left(t_{i+1}\right) & =f_{\Lambda_{i+1}^{-1}\left(X_{i+1}+\Lambda_{i+1}\left(t_{i}\right)\right)}\left(t_{i+1}\right) \\
& =\lambda_{i+1}\left(t_{i+1}\right) f_{X_{i+1}}\left(\Lambda_{i+1}\left(t_{i+1}\right)-\Lambda_{i+1}\left(t_{i}\right)\right),
\end{aligned}
$$

which easily provides the result, after substitution into (17).

In the next result, we present some dependence properties for a trendtransformed independent vector. Let us recall that the concept of Multivariate Total Positivity of order 2 (MTP2) is one of the strongest concept of positive dependence, which implies, in particular, the Conditionally Increasingness in Sequence (CIS). The negative counterpart of this property is the Conditionally Decreasingness in Sequence (CDS). A thorough study of MTP2 random vectors is given in [Karlin and Rinott(1980)]. The definitions for the other multivariate dependence concepts can be found in [Müller and Stoyan(2002), Ch.3.10], for instance.

In order to derive the multivariate results below, we need conditions expressed through aging properties of univariate random variables. We recall that a univariate random variable is said to be Increasing Failure Rate (IFR) when its survival function is log-concave and Decreasing Failure Rate (DFR) when it is log-convex. These classical reliability concepts can be found in many text books 
of applied probability and reliability (see, [Müller and Stoyan(2002), p.46] for instance). Recall from Remark 1 that a trend function $\Lambda$ can be seen as the cumulative hazard rate of a univariate random variable $Y$. Then, aging properties of this random variable can be translated into shape properties for $\Lambda$. In particular, $Y$ is IFR (DFR) if and only if $\Lambda$ is concave (convex).

Proposition 12 Let $\left(T_{1}, \ldots, T_{n}\right)$ be a trend-transformed independent vector with trend function $\boldsymbol{\Lambda}=\left(\Lambda_{1}, \ldots, \Lambda_{n}\right)$ and increment distributions $\mathbf{F}=\left(F_{1}, \ldots, F_{n}\right)$.

(a) Assume that each $\Lambda_{i}$ is absolutely continuous with corresponding intensity function $\lambda_{i}$ and that each $F_{i}$ admits a density $f_{i}$. If $f_{i}$ are log-concave functions, $i=1,2 \ldots, n$, then $\left(T_{1}, \ldots, T_{n}\right)$ is MTP2;

(b) Let $Y_{i}$ be a random variable with cumulative hazard rate $\Lambda_{i}, i=1, \ldots, n$. Let $\left(W_{1}, \ldots, W_{n}\right)$ be the vector of inter-arrival times, where $W_{i}=T_{i}-$ $T_{i-1}, i=1, \ldots, n$ (with $W_{0}=0$ ). Then, if each $Y_{i}$ is DFR (IFR), $i=$ $1,2 \ldots, n$, the vector $\left(W_{1}, \ldots, W_{n}\right)$ is $C I S(C D S)$.

\section{Proof:}

(a) The proof is similar to [Badía et al(2018)b, Thm. 1(a)]. Recall by (11) that the joint density function of $\left(T_{1}, \ldots, T_{n}\right)$ is given by

$$
f_{\left(T_{1}, \ldots, T_{n}\right)}\left(t_{1}, \ldots, t_{n}\right)=\prod_{i=1}^{n} \lambda_{i}\left(t_{i}\right) \prod_{i=1}^{n} f_{i}\left(\Lambda_{i}\left(t_{i}\right)-\Lambda_{i}\left(t_{i-1}\right)\right) 1_{A}\left(t_{1}, \ldots, t_{n}\right)
$$

(0 otherwise), where $A:=\left\{\left(t_{1}, \ldots, t_{n}\right) \in \mathbb{R}^{n} \mid-\infty=t_{0}<t_{1}<t_{2}<\cdots<\right.$ $\left.t_{n}, t_{i}<M_{i}, i=1, \ldots, n\right\}$

As the product of MTP2 functions is also MTP2 [Karlin and Rinott(1980), Prop. 3.3], it is sufficient to check this property for each term. First of all, $1_{A}$ is MTP2, because $A$ is a lattice (closed under minimum and maximum). The terms $\lambda_{i}\left(t_{i}\right)$ and $f_{1}\left(\Lambda_{1}\left(t_{1}\right)\right.$ (case $j=1$ in the previous product) are also MTP2 because they are unidimensional. Thus, it only remains to check the MTP2 property for:

$$
g\left(t_{1}, \ldots, t_{n}\right)=f_{i}\left(\Lambda_{i}\left(t_{i}\right)-\Lambda_{i}\left(t_{i-1}\right)\right), \quad j=2, \ldots, n .
$$

As $f_{i}$ is log-concave, $f_{i}(x-y)$ is TP2 (see [Marshall and Olkin (2007), p. 696]) and thus $g^{*}\left(t_{1}, \ldots, t_{n}\right)=\prod_{j=2}^{n} f_{i}\left(t_{j}-t_{j-1}\right)$ is MTP2. The MTP2 for $g$ now follows because $\Lambda_{i}$ is increasing and this property is preserved by increasing transforms ([Karlin and Rinott(1980), Prop. 3.6]).

(b) The proof follows the ideas of [Badía et al(2018)b, Thm. 1(c)], but we provide the details here. Based on (3), the properties of the inverse function and straightforward algebra, we have

$$
\begin{aligned}
& \mathbb{P}\left(W_{i+1}>x \mid W_{1}=w_{1}, \ldots, W_{i}=w_{i}\right) \\
& =\mathbb{P}\left(T_{i+1}>x+T_{i} \mid W_{1}=w_{1}, \ldots, W_{i}=w_{i}\right)
\end{aligned}
$$




$$
\begin{aligned}
& =\mathbb{P}\left(\Lambda_{i+1}^{-1}\left(X_{i+1}+\Lambda_{i+1}\left(T_{i}\right)\right)>x+T_{i} \mid W_{1}=w_{1}, \ldots, W_{i}=w_{i}\right) \\
& =\mathbb{P}\left(X_{i+1}+\Lambda_{i+1}\left(T_{i}\right)>\Lambda_{i+1}\left(x+T_{i}\right) \mid W_{1}=w_{1}, \ldots, W_{i}=w_{i}\right) \\
& =\mathbb{P}\left(X_{i+1}>\Lambda_{i+1}\left(x+T_{i}\right)-\Lambda_{i+1}\left(T_{i}\right) \mid W_{1}=w_{1}, \ldots, W_{i}=w_{i}\right) \\
& =\bar{F}_{i+1}\left(\Lambda_{i+1}\left(x+\sum_{j=1}^{i} w_{j}\right)-\Lambda_{i+1}\left(\sum_{j=1}^{i} w_{j}\right)\right)
\end{aligned}
$$

for all $i=1, \ldots, n-1$. Now, assuming that $Y_{i+1}$ is DFR (IFR), its survival function is log-convex (log-concave) and its cumulative hazard rate is concave (convex). Then, $\Lambda_{i+1}\left(x+\sum_{j=1}^{i} w_{j}\right)-\Lambda_{i+1}\left(\sum_{j=1}^{i} w_{j}\right)$ is decreasing (increasing) in $\left(w_{1}, \ldots, w_{i}\right)$. The conclusion follows as $\bar{F}_{i+1}$ is decreasing.

Remark 5 Property (b) in the previous result, applied in the setting of Example 8 provides us with results concerning dependence properties of spacings in either sequential or generalized order statistics. As said before, the proof of the previous proposition is based on [Badía et al(2018)b, Thm. 1], thus extending several dependence properties for a trend renewal process to trend-transformed independent vectors. Moreover, in [Badía et al(2018)b, Thm. 1] a result concerning the multivariate increasing failure rate property for the vector of arrival times in a trend renewal process is provided. This result can also be generalized to trend-transformed independent vectors. Details are omitted, as the definitions and results are quite technical. Besides, the previous result includes some known properties for Generalized Pòlya Processes (see [Badía et al(2018a), Prop. 10 and 11]).

Remembering that a trend function $\Lambda$ can be seen as the cumulative hazard rate of a random variable $Y$, many multivariate stochastic comparison results previously obtained in the literature for specific $\left(T_{1}, \ldots, T_{n}\right)$ (as described in Examples 4, 5, 8 or 9) rely on comparison assumptions on the corresponding random variable $Y$ (see, e.g. [Belzunce et al(2001), Zhuang et al(2007)]). In this way, hazard rate comparisons are used between such random variables in different models in order to obtain, for instance, stochastic comparisons for general sequential order statistics or non-homogeneous pure-birth processes. However for some specific models (GOS [Belzunce et al(2005)], or trend renewal processes [Badía et al(2018)b], for instance), these conditions can be weaken to comparison assumptions in the usual stochastic order. Our aim here is to give a general result including both cases. To begin with, we give a new characterization of the hazard rate ordering, which is adapted to the construction of $\left(T_{1}, \ldots, T_{n}\right)$, as provided by (3). Recall that two random variables $Y_{1}$ and $Y_{2}$ with respective survival functions $\bar{F}_{1}$ and $\bar{F}_{2}$ are ordered in the hazard rate order if and only if $\bar{F}_{2} / \bar{F}_{1}$ is an increasing function (see [Müller and Stoyan(2002), p.8], for instance).

Proposition 13 Let $\Lambda$ and $\widetilde{\Lambda}$ be two trend functions with respective endpoints $M^{(j)}, j=1,2$, and such that $M^{(1)} \leq M^{(2)}$. Let us introduce two random 
variables $Y^{(j)}, j=1,2$ with respective cumulative hazard rates $\Lambda$ and $\widetilde{\Lambda}$. Then, we have the following equivalence: $Y^{(1)} \leq_{h r} Y^{(2)}$ if and only if

$$
\Lambda^{-1}(x+\Lambda(y)) \leq \widetilde{\Lambda}^{-1}(x+\widetilde{\Lambda}(y)), \quad x>0, y \leq M^{(1)} .
$$

Proof. First, we recall from (1) that the survival function of a random variable with cumulative hazard rate $\Lambda$ can be written as $e^{-\Lambda(x)}$ and therefore, it is easy to check from the definition of hazard rate order that

$$
\begin{aligned}
Y^{(1)} \leq_{h r} Y^{(2)} & \Leftrightarrow e^{-(\widetilde{\Lambda}-\Lambda)} \text { is an increasing function on }\left(-\infty, M^{(1)}\right) \\
& \Leftrightarrow \widetilde{\Lambda}-\Lambda \text { is a decreasing function on }\left(-\infty, M^{(1)}\right) .
\end{aligned}
$$

Now, assume that $Y^{(1)} \leq_{h r} Y^{(2)}$, so that $\widetilde{\Lambda}-\Lambda$ is a decreasing function. Our aim is to prove (18). To begin with, let us notice that

$$
\widetilde{\Lambda}^{-1}(x+\widetilde{\Lambda}(y))>y \quad x>0, y \leq M^{(2)} .
$$

This is because

$$
\widetilde{\Lambda}\left(\widetilde{\Lambda}^{-1}(x+\widetilde{\Lambda}(y))=x+\widetilde{\Lambda}(y)>\widetilde{\Lambda}(y),\right.
$$

which clearly implies (20). From now on, assume that $\widetilde{\Lambda}^{-1}(x+\widetilde{\Lambda}(y)) \leq M^{(1)}$ (otherwise (18) holds trivially as the first term is less or equal that $M^{(1)}$ ). Based on the fact that $\widetilde{\Lambda}-\Lambda$ is a decreasing function and $\widetilde{\Lambda}\left(\widetilde{\Lambda}^{-1}(s)\right)=s$ for all $s>0$, we have

$$
x=\widetilde{\Lambda}\left(\widetilde{\Lambda}^{-1}(x+\widetilde{\Lambda}(y))\right)-\widetilde{\Lambda}(y) \leq \Lambda\left(\widetilde{\Lambda}^{-1}(x+\widetilde{\Lambda}(y))\right)-\Lambda(y)
$$

and therefore

$$
x+\Lambda(y) \leq \Lambda\left(\widetilde{\Lambda}^{-1}(x+\widetilde{\Lambda}(y))\right) .
$$

Now let us denote $D$ and $\widetilde{D}$ the sets defined in (5). Note that $D \subseteq \widetilde{D}$ (as on an interval $I_{j} \subseteq D, \widetilde{\Lambda}$ has to be constant too, because otherwise $\widetilde{\Lambda}-\Lambda$ would not be decreasing, thus $I_{j} \subseteq D \subseteq \widetilde{D}$ ). Therefore, $\widetilde{D}^{c} \subseteq D^{c}$. Applying $\Lambda^{-1}$ to both terms of (21) and taking into account that $\left.\widetilde{\Lambda}^{-1}(x+\widetilde{\Lambda}(y))\right) \in D^{c}$, we obtain

$$
\Lambda^{-1}(x+\Lambda(y)) \leq \Lambda^{-1}\left(\Lambda\left(\widetilde{\Lambda}^{-1}(x+\widetilde{\Lambda}(y))\right)\right)=\widetilde{\Lambda}^{-1}(x+\widetilde{\Lambda}(y)),
$$

which completes the proof of the "if" part. For the "only if" part, assume (18) to hold. Based on (19), the point is to show that $\widetilde{\Lambda}-\Lambda$ is a decreasing function on $\left(-\infty, M^{(1)}\right)$, that is

$$
\widetilde{\Lambda}(y)-\Lambda(y) \leq \widetilde{\Lambda}(x)-\Lambda(x), \quad x<y \leq M^{(1)} .
$$

Let $x<y \leq M^{(1)}$. If $\widetilde{\Lambda}(y)=\widetilde{\Lambda}(x)$, the result is clear. Now, assume that $\widetilde{\Lambda}(y)>$ $\widetilde{\Lambda}(x)$. If $y \in \widetilde{I}_{j}=\left(a_{j}, b_{j}\right] \subseteq \widetilde{D}$, then $x<a_{j}$, because $\widetilde{\Lambda}\left(a_{j}\right)=\widetilde{\Lambda}(y)>\widetilde{\Lambda}(x)$. Note that we have

$$
\widetilde{\Lambda}(y)-\Lambda(y) \leq \widetilde{\Lambda}\left(a_{j}\right)-\Lambda\left(a_{j}\right) .
$$


Hence, it is enough to show that

$$
\widetilde{\Lambda}\left(a_{j}\right)-\Lambda\left(a_{j}\right) \leq \widetilde{\Lambda}(x)-\Lambda(x),
$$

with $x<a_{j}$ and $a_{j} \in \widetilde{D}^{c}$ to conclude. Clearly, this is true if we know that (22) is true for all $y \in \widetilde{D}^{c}$. Then we can reduce the study to this case. From now on, we hence assume that $y \in \widetilde{D}^{c}$ and $\widetilde{\Lambda}(y)>\widetilde{\Lambda}(x)$. Applying (18) with $x^{\star}=\widetilde{\Lambda}(y)-\widetilde{\Lambda}(x)(>0)$ and $y^{\star}=x$, we obtain

$$
y=\widetilde{\Lambda}^{-1}(\widetilde{\Lambda}(y))=\widetilde{\Lambda}^{-1}(\widetilde{\Lambda}(y)-\widetilde{\Lambda}(x)+\widetilde{\Lambda}(x)) \geq \Lambda^{-1}(\widetilde{\Lambda}(y)-\widetilde{\Lambda}(x)+\Lambda(x)) .
$$

Now, (22) is derived by applying $\Lambda$ in both terms.

In the following result, we give conditions for comparison in the multivariate stochastic ordering.

Proposition 14 Let us consider two trend-transformed independent vectors $\left(T_{1}^{(j)}, \ldots, T_{n}^{(j)}\right), j=1,2$ with respective trend vectors $\boldsymbol{\Lambda}$ (with endpoint vector $\left.\mathbf{M}^{(1)}\right)$ and $\widetilde{\boldsymbol{\Lambda}}$ (with endpoint vector $\mathbf{M}^{(2)}$ ) and increment distributions $\left(X_{1}^{(j)}, \ldots, X_{n}^{(j)}\right), j=$ 1,2. Assume that for each $i=1, \ldots, n$, we have $M_{i}^{(1)} \leq M_{i}^{(2)}, X_{i}^{(1)} \leq_{s t} X_{i}^{(2)}$ and $\Lambda_{i} \geq \widetilde{\Lambda}_{i}$ (on $\left(-\infty, M_{i}^{(1)}\right)$. Moreover, assume that there exists $1 \leq n^{\prime} \leq n$ such that

$$
\begin{aligned}
& \Lambda_{i} \circ \Lambda_{i-1}^{-1} \leq \widetilde{\Lambda}_{i} \circ \widetilde{\Lambda}_{i-1}^{-1} \text { for } 2 \leq i \leq n^{\prime}, \\
& \widetilde{\Lambda}_{i}-\Lambda_{i} \text { is decreasing on }\left(-\infty, M_{i}^{(1)}\right) \text { for } n^{\prime}<i \leq n .
\end{aligned}
$$

Then,

$$
\left(T_{1}^{(1)}, \ldots, T_{n}^{(1)}\right) \leq_{s t}\left(T_{1}^{(2)}, \ldots, T_{n}^{(2)}\right)
$$

Also, it is possible to construct the two vectors $\left(T_{1}^{(j)}, \ldots, T_{n}^{(j)}\right), j=1,2$ on the same probability space in such a way that

$$
\left(T_{1}^{(1)}, \cdots, T_{n}^{(1)}\right) \leq\left(T_{1}^{(2)}, \cdots, T_{n}^{(2)}\right) \quad \text { a.s. }
$$

Proof. It is enough to show the second result, as the almost sure order implies the usual stochastic one. On one hand, the ordering condition for the vectors $\left(X_{1}^{(j)}, \ldots, X_{n}^{(j)}\right), j=1,2$ allows us to construct the two vectors $\left(X_{1}^{(j)}, \ldots, X_{n}^{(j)}\right), j=1,2$ on the same probability space in such a way that

$$
\left(X_{1}^{(1)}, \cdots, X_{n}^{(1)}\right) \leq\left(X_{1}^{(2)}, \cdots, X_{n}^{(2)}\right) \text { a.s. }
$$

On the other hand, the two vectors $\left(T_{1}^{(j)}, \ldots, T_{n}^{(j)}\right), j=1,2$ can be defined in this probability space using (3), which is also valid a.s. The case $i=1$ is then obvious, as $\Lambda_{1} \geq \widetilde{\Lambda}_{1}$ implies $\Lambda_{1}^{-1} \leq \widetilde{\Lambda}_{1}^{-1}$, and therefore

$$
T_{1}^{(1)}=\Lambda_{1}^{-1}\left(X_{1}^{(1)}\right) \leq \widetilde{\Lambda}_{1}^{-1}\left(X_{1}^{(2)}\right)=T_{1}^{(2)} .
$$


Now, based on (3), we have

$$
\begin{aligned}
& T_{2}^{(1)}=\Lambda_{2}^{-1}\left(X_{2}^{(1)}+\left(\Lambda_{2} \circ \Lambda_{1}^{-1}\right)\left(X_{1}^{(1)}\right)\right), \\
& T_{3}^{(1)}=\Lambda_{3}^{-1}\left(X_{3}^{(1)}+\left(\Lambda_{3} \circ \Lambda_{2}^{-1}\right)\left(X_{2}^{(1)}+\left(\Lambda_{2} \circ \Lambda_{1}^{-1}\right)\left(X_{1}^{(1)}\right)\right)\right) .
\end{aligned}
$$

Clearly, any $T_{i}^{(1)}$ can be expressed in a similar way, with

$T_{i}^{(1)}=\Lambda_{i}^{-1}\left(X_{i}^{(1)}+\left(\Lambda_{i} \circ \Lambda_{i-1}^{-1}\right)\left(X_{i-1}^{(1)}+\left(\Lambda_{i-1} \circ \Lambda_{i-2}^{-1}\right)\left(\ldots+\left(\Lambda_{2} \circ \Lambda_{1}^{-1}\right)\left(X_{1}^{(1)}\right)\right)\right)\right)$,

where only $\Lambda_{i}^{-1}$ and $\Lambda_{k} \circ \Lambda_{k-1}^{-1}, k=2, \ldots, i$ are involved, with a similar expression for $T_{i}^{(2)}$ with respect to $\widetilde{\Lambda}_{i}^{-1}$ and $\widetilde{\Lambda}_{k} \circ \widetilde{\Lambda}_{k-1}^{-1}, k=2, \ldots, i$.

Let $n^{\prime}$ be as in (23). Using that $\Lambda_{i} \circ \Lambda_{i-1}^{-1} \leq \widetilde{\Lambda}_{i} \circ \widetilde{\Lambda}_{i-1}^{-1}$, it is easy to derive from the previous expressions that $T_{i}^{(1)} \leq T_{i}^{(2)}$ (a.s.). Thus, this inequality is true up to $n^{\prime}$.

For $i \geq n^{\prime}$, we proceed by induction, where the case $i=n^{\prime}$ is already known to be true. Let $i$ be such that $n^{\prime} \leq i \leq n-1$ and assume that $T_{i}^{(1)} \leq T_{i}^{(2)}$. Let $Y_{i+1}^{(j)}, j=1,2$ be two random variables with respective cumulative hazard rate functions $\Lambda_{i+1}$ and $\widetilde{\Lambda}_{i+1}$. Condition (24) is equivalent to say that $Y_{i+1}^{(1)} \leq_{h r} Y_{i+1}^{(2)}$ (recall (19)). Thus, applying Proposition 13, we derive that

$$
\begin{gathered}
\left.T_{i+1}^{(1)}=\Lambda_{i+1}^{-1}\left(X_{i+1}^{(1)}+\Lambda_{i+1}\left(T_{i}^{(1)}\right)\right) \leq \widetilde{\Lambda}_{i+1}^{-1}\left(X_{i+1}^{(1)}+\widetilde{\Lambda}_{i+1}\left(T_{i}^{(1)}\right)\right)\right) \\
\leq \widetilde{\Lambda}_{i+1}^{-1}\left(X_{i+1}^{(2)}+\widetilde{\Lambda}_{i+1}\left(T_{i}^{(2)}\right)\right)=T_{i+1}^{(2)}
\end{gathered}
$$

We then conclude that $T_{i}^{(1)} \leq T_{i}^{(2)}$ for $1 \leq i \leq n$, and thus (26) holds.

Remark 6 (a) If $\Lambda_{i}=\Lambda$ and $\widetilde{\Lambda}_{i}=\widetilde{\Lambda}, i=1,2, \ldots n$, we recall that $\left(\Lambda \circ \Lambda^{-1}\right)(s)=$ $\left(\widetilde{\Lambda} \circ \widetilde{\Lambda}^{-1}\right)(s)=s, s>0$ and (23) in the previous result holds true trivially (with $n^{\prime}=n$ ). Thus, in this case the problem reduces to check that $M^{(1)} \leq M^{(2)}, \Lambda \geq \widetilde{\Lambda}$ and $X_{i}^{(1)} \leq_{s t} X_{i}^{(2)}$ for each $i=1, \ldots, n$. These conditions are the same as the ones given in [Badía et al(2018)b, Thm. 3(a)] for trend renewal processes.

(b) Let $h^{(j)}:\{0,1, \ldots, n-1\} \rightarrow \mathbb{R}_{+}^{*}$ be given functions and let $F_{i}^{(j)}$ stand for the exponential distribution function with mean $1 / h^{(j)}(i-1)$, with $i=1, \ldots, n$ and $j=1,2$. Assume further that $\Lambda_{i}=\Lambda$ and $\widetilde{\Lambda}_{i}=\widetilde{\Lambda}, i=1,2, \ldots n$. Based on point (a), the problem reduces to check that $M^{(1)} \leq M^{(2)}, \Lambda \geq \widetilde{\Lambda}$ and $h^{(1)} \geq h^{(2)}$. Hence, the previous result extends in a natural way those obtained in Belzunce et al. [Belzunce et al(2005), Thm. 3.10(i)] for Generalized Order Statistics (see Example 8) and in Badía et al [Badía et al(2018c), Lemma 2] for the arrival times of an Extended Pólya process (see Example $5)$. 
(c) If, for instance, $\Lambda_{i}=\widetilde{\Lambda}_{i}, i=1, \ldots, n^{\prime}$, but $\Lambda_{n^{\prime}+1} \neq \widetilde{\Lambda}_{n^{\prime}+1}$, then condition (23) is contradictory with $\Lambda_{n^{\prime}+1} \geq \widetilde{\Lambda}_{n^{\prime}+1}$. Thus, condition (24) could be helpful in these situations. The general results for multivariate stochastic order for non-homogeneous pure-birth processes [Belzunce et al(2001), Thm.3.11] or for sequential order statistics [Zhuang et al(2007), Thm. 3.7] were given under similar conditions with $n^{\prime}=2$. Thus, our previous proposition enlarges such results.

(d) In view of Examples (a) and (b), it might be tempting to think that the conditions $M_{i}^{(1)} \leq M_{i}^{(2)}, X_{i}^{(1)} \leq_{s t} X_{i}^{(2)}$ and $\Lambda_{i} \geq \widetilde{\Lambda}_{i}$ could be sufficient for the stochastic order to hold. But it is easy to construct a counterexample as follows. Let $\Lambda_{1}(x)=\widetilde{\Lambda}_{1}(x)=x, x>0$. Assume that $\widetilde{\Lambda}_{2}$ and $\Lambda_{2}$ are equal to 0 on $(-\infty, 0]$ such that $M_{i}^{(1)}=M_{i}^{(2)}=\infty$ for each $i$ and that $\widetilde{\Lambda}_{2}-\Lambda_{2}$ is not a decreasing function. According to Proposition 13, there exists $x, y>0$ such that

$$
\Lambda_{2}^{-1}\left(x+\Lambda_{2}(y)\right)>\widetilde{\Lambda}_{2}^{-1}\left(x+\widetilde{\Lambda}_{2}(y)\right) .
$$

Take two independent uniform random variables $U_{1}$ and $U_{2}$ (on the same probability space), and consider $X_{1}^{(1)}=X_{1}^{(2)}=y-h U_{1}$ and $X_{2}^{(1)}=X_{2}^{(2)}=$ $x-h U_{2}$ (h small enough). Let us next set

$T_{2}^{(1)}=\Lambda_{2}^{-1}\left(x-h U_{2}+\Lambda_{2}\left(y-h U_{1}\right)\right)$ and $T_{2}^{(2)}=\widetilde{\Lambda}_{2}^{-1}\left(x-h U_{2}+\widetilde{\Lambda}_{2}\left(y-h U_{1}\right)\right)$.

If $T_{2}^{(1)} \leq T_{2}^{(2)}$ a.s. for all $h>0$, taking limits as $h \downarrow 0$ (recall that the inverse functions are left-continuous) leads to a contradiction with (28). Hence, $T_{2}^{(1)} \leq T_{2}^{(2)}$ cannot be almost surely true for all $h>0$.

In the following corollary, we next express the previous conditions from Proposition 14 for sequential and intermediate order statistics, in terms of their associated distribution functions.

Corollary 15 We have the following.

(a) Let $\left(\bar{G}_{1, j}, \ldots, \bar{G}_{n, j}\right), j=1,2$ be two vectors of survival functions such that $M_{1, j}=\left(\bar{G}_{1, j}\right)^{-1}(0) \leq \ldots \leq M_{n, j}=\left(\bar{G}_{n, j}\right)^{-1}(0)$ for $j=1,2$, and let $\left(X_{1, n}^{\star, j}, \ldots, X_{n, n}^{\star, j}\right), j=1,2$ be the sequential order statistics for the corresponding distribution functions (see Example 8). If we have $M_{i, 1} \leq M_{i, 2}$, $\bar{G}_{i, 2} \geq \bar{G}_{i, 1}$ for all $i=1, \ldots, n$ and if there exists some $1 \leq n^{\prime} \leq n$ such that:

(i) there exist two vectors of positive real numbers $\left(r_{1, j}, \ldots, r_{n^{\prime}, j}\right), j=1,2$ such that $r_{i, 1} \geq r_{i, 2}$ for all $i=1, \ldots, n$ and $\bar{G}_{i, 1}^{r_{i, 1}} \circ\left(\bar{G}_{i-1,1}^{r_{i-1,1}}\right)^{-1} \leq$ $\bar{G}_{i, 2}^{r_{i, 2}} \circ\left(\bar{G}_{i-1,2}^{r_{i-1,2}}\right)^{-1}$ for $2 \leq i \leq n^{\prime}$,

(ii) $\bar{G}_{i, 1} \leq_{h r} \bar{G}_{i, 2}$ for $n^{\prime}<i \leq n$ (where the hazard rate order stands for the corresponding random variables), 
then $\left(X_{1, n}^{\star, 1}, \ldots, X_{n, n}^{\star, 1}\right) \leq_{s t}\left(X_{1, n}^{\star, 2}, \ldots, X_{n, n}^{\star, 2}\right)$

(b) Let $G_{1}$ and $G_{2}$ be two distribution functions and let $\left(W_{1}^{(j)}, \ldots, W_{n}^{(j)}\right), j=$ 1,2 be the associate vector of intermediate order statistics of order $0=$ $p_{0}^{(j)} \leq p_{1}^{(j)} \leq p_{2}^{(j)} \leq \cdots \leq p_{n}^{(j)} \leq n$ (see Example 10). If $G_{1} \geq G_{2}$ and $p_{i}^{(1)}-$ $p_{i-1}^{(1)} \leq p_{i}^{(2)}-p_{i-1}^{(2)}, i=1 \ldots n$, then $\left(W_{1}^{(1)}, \ldots, W_{n}^{(1)}\right) \leq_{s t}\left(W_{1}^{(2)}, \ldots, W_{n}^{(2)}\right)$.

Proof. For point (a), let us first remember from Example 8 that sequential order statistics are trend-transformed independent vectors with trend functions $\Lambda_{i, j}, 1 \leq i \leq n, j=1,2$, defined as the cumulative hazard rates of the $\bar{G}_{i, j}$ 's and exponential increments with rate 1 . Alternatively, we can also consider $\hat{\Lambda}_{i, j}=$ $r_{i, j} \Lambda_{i, j}$ and exponential random variables $\hat{X}_{i, j}$ with means $1 / r_{i, j}$ (see Remark $3)$. Our aim is to show that the conditions from Proposition 14 are true for this last parameterization. As $r_{i, 1} \geq r_{i, 2}$, we already know that $\hat{X}_{i, 1} \leq$ sto $\hat{X}_{i, 2}$. Also, based on $\Lambda_{i, j}=-\ln \circ \bar{G}_{i, j}$, it is clear that $\Lambda_{i, 1} \geq \Lambda_{i, 2}$ due to $\bar{G}_{i, 2} \geq \bar{G}_{i, 1}$ by assumption. This implies that $\hat{\Lambda}_{i, 1} \geq \hat{\Lambda}_{i, 2}$ because $r_{i, 1} \geq r_{i, 2}$. For $2 \leq i \leq n^{\prime}$, let us show (23), namely

$$
\hat{\Lambda}_{i, 1} \circ\left(\hat{\Lambda}_{i-1,1}\right)^{-1} \leq \hat{\Lambda}_{i, 2} \circ\left(\hat{\Lambda}_{i-1,2}\right)^{-1}
$$

We have

$$
\begin{aligned}
\hat{\Lambda}_{i, j} \circ\left(\hat{\Lambda}_{i-1, j}\right)^{-1} & =r_{i, j} \Lambda_{i, j} \circ\left(r_{i-1, j} \Lambda_{i-1, j}\right)^{-1} \\
& =-r_{i, j} \ln \circ \bar{G}_{i, j} \circ\left(-r_{i-1, j} \ln \circ \bar{G}_{i-1, j}\right)^{-1} \\
& =-\ln \circ \bar{G}_{i, j}^{r_{i, j}} \circ\left(-\ln \circ \bar{G}_{i-1, j}^{r_{i-1, j}}\right)^{-1} .
\end{aligned}
$$

Based on

$$
\left(\left(-\ln \circ \bar{G}_{i-1, j}^{r_{i-1, j}}\right)^{-1}\right)(t)=\left(\left(\bar{G}_{i-1, j}^{r_{i-1, j}}\right)^{-1} \circ(-\ln )^{-1}\right)(t)=\left(\bar{G}_{i-1, j}^{r_{i-1, j}}\right)^{-1}\left(e^{-t}\right)
$$

for all $t \geq 0$ (see [Embrechts and Hofert(2013)] for the first equality), we now get

$$
\left(\hat{\Lambda}_{i, j} \circ\left(\hat{\Lambda}_{i-1, j}\right)^{-1}\right)(t)=-\left(\ln \circ \bar{G}_{i, j}^{r_{i, j}} \circ\left(\bar{G}_{i-1, j}^{r_{i-1, j}}\right)^{-1}\right)\left(e^{-t}\right), \quad t \geq 0
$$

from where (29) is easily derived, based on the assumptions. Now, for $n^{\prime}<i \leq$ $n$, as $\bar{G}_{i, 1} \leq_{h r} \bar{G}_{i, 2}$, we know from (19) that $\Lambda_{i, 2}-\Lambda_{i, 1}$ decreases and hence,

$$
\hat{\Lambda}_{i, 2}-\hat{\Lambda}_{i, 1}=r_{i, 2}\left(\Lambda_{i, 2}-\Lambda_{i, 1}\right)-\left(r_{i, 1}-r_{i, 2}\right) \Lambda_{i, 1}
$$

also decreases and (24) is true. This completes the proof of point (a). For point (b), let $\Lambda_{j}$ be the cumulative hazard rate for $G_{j}, j=1,2$. We recall from Example 10 that intermediate order statistics are trend-transformed independent vectors with trend vector $\left(\Lambda_{1, j}=\Lambda_{j}, \ldots, \Lambda_{n, j}=\Lambda_{j}\right)$ and increment distributions defined as $X_{i, j}=-\ln B_{i, j}, i=1, \ldots, n, j=1,2$, where $B_{i, j}$ is a beta 
random variable with parameters $a=n-p_{i}^{(j)}+1$ and $b=p_{i}^{(j)}-p_{i-1}^{(j)}$. As $\Lambda_{j}=-\ln \left(1-G_{j}\right)$, we have $\Lambda_{1} \geq \Lambda_{2}$. Based on Remark 6(a), conditions (23) and (24) hence hold and there only remains to check that $X_{i, 1} \leq_{s t o} X_{i, 2}$. We recall that for beta random variables

$$
B(a, b) \leq_{s t} B\left(a^{\star}, b^{\star}\right) \quad \text { if } \quad a \leq a^{\star} \text { and } b \geq b^{\star},
$$

and therefore, as $-\ln (\cdot)$ is a decreasing function,

$$
-\ln B(a, b) \leq_{s t}-\ln B\left(a^{\star}, b^{\star}\right) \quad \text { if } \quad a \geq a^{\star} \text { and } b \leq b^{\star} .
$$

In our setting, sufficient conditions hence are:

$$
n-p_{i}^{(1)}+1 \geq n-p_{i}^{(2)}+1, \text { and } p_{i}^{(1)}-p_{i-1}^{(1)} \leq p_{i}^{(2)}-p_{i-1}^{(2)}, \quad i=1, \ldots, n .
$$

Under the second condition, we have

$$
p_{i}^{(1)}=\sum_{k=1}^{i}\left(p_{k}^{(1)}-p_{k-1}^{(1)}\right) \leq \sum_{k=1}^{i}\left(p_{k}^{(2)}-p_{k-1}^{(2)}\right)=p_{i}^{(2)}, i=1,2, \ldots, n,
$$

which implies the first condition. The second condition is hence sufficient to ensure that $X_{i, 1} \leq_{\text {sto }} X_{i, 2}$, which completes the proof.

We now provide comparison results in the likelihood ratio ordering. Recall that two random variables $Y_{1}$ and $Y_{2}$ with respective density functions $\bar{f}_{1}$ and $\bar{f}_{2}$ are ordered in the likelihood ratio ordering if and only if $\bar{f}_{2} / \bar{f}_{1}$ is an increasing function (see [Müller and Stoyan(2002), p.12], for instance). We are going to use the multivariate generalization of this order (see [Müller and Stoyan(2002), p.129], for instance), whose definition is provided in (41). In order to obtain as accurate as possible conditions, we consider a specific case where the increments are identically gamma distributed, with identical parameters for the two trendtransformed independent vectors to be compared.

Theorem 16 Let us consider two trend-transformed independent vectors, $\left(T_{1}^{(j)}, \ldots, T_{n}^{(j)}\right)$, $j=1,2$ with respective trend vectors $\Lambda^{(\mathbf{j})}, j=1,2$, which are assumed to be absolutely continuous with respective intensity functions $\lambda^{(\mathbf{j})}, j=1,2$ and endpoints $\mathbf{M}^{(j)}, j=1,2$. Assume that $M_{i}^{(1)} \leq M_{i}^{(2)}$ for each $i=1, \ldots, n$, and that the vectors of increment distribution $\left(X_{1}^{(j)}, \ldots, X_{n}^{(j)}\right), j=1,2$ are identically distributed, and such that each $X_{i}^{(j)}$ has the following gamma density:

$$
f_{i}(x)=\frac{\alpha^{p} x^{p-1}}{\Gamma(p)} e^{-\alpha x}, \quad x>0, \alpha>0 \text { and } p \geq 1 .
$$

Let $Y_{i}^{(j)}$ be random variables with intensity functions $\lambda_{i}^{(j)}, i=1, \ldots, n, j=1,2$. Assume that the following conditions hold:

(a) $Y_{i}^{(1)} \leq_{h r} Y_{i}^{(2)}, i=1, \ldots, n$, 
(b) $\lambda_{i}^{(2)} / \lambda_{i}^{(1)}$ is increasing, $i=1, \ldots, n$,

(c) $\Lambda_{i}^{(1)}-\Lambda_{i+1}^{(1)}-\Lambda_{i}^{(2)}+\Lambda_{i+1}^{(2)}$ is increasing, $i=1, \ldots, n-1$.

Then, $\left(T_{1}^{(1)}, \ldots, T_{n}^{(1)}\right) \leq_{l r}\left(T_{1}^{(2)}, \ldots, T_{n}^{(2)}\right)$.

Proof. Let $\mathbf{f}^{(j)}$ be the joint density functions corresponding to each $\left(T_{1}^{(j)}, \ldots, T_{n}^{(j)}\right)$, $j=1,2$. Let $\mathbf{x}$ and $\mathbf{y}$ be two vectors in $\mathbb{R}^{n}$. In order to check the likelihood ratio order, we need to prove that

$$
\mathbf{f}^{(1)}(\mathbf{x}) \mathbf{f}^{(2)}(\mathbf{y}) \leq \mathbf{f}^{(1)}(\mathbf{x} \wedge \mathbf{y}) \mathbf{f}^{(2)}(\mathbf{x} \vee \mathbf{y}) .
$$

According to Proposition 11, the product of the two joint pdf can be written as

$$
\begin{gathered}
\mathbf{f}^{(1)}(\mathbf{x}) \mathbf{f}^{(2)}(\mathbf{y})=C \prod_{i=1}^{n} \lambda_{i}^{(1)}\left(x_{i}\right) \lambda_{i}^{(2)}\left(y_{i}\right) \times e^{-\alpha \Lambda_{n}^{(1)}\left(x_{n}\right)} e^{-\alpha \Lambda_{n}^{(2)}\left(y_{n}\right)} \\
\times \prod_{i=1}^{n-1} e^{-\alpha\left(\Lambda_{i}^{(1)}\left(x_{i}\right)-\Lambda_{i+1}^{(1)}\left(x_{i}\right)\right)} e^{-\alpha\left(\Lambda_{i}^{(2)}\left(y_{i}\right)-\Lambda_{i+1}^{(2)}\left(y_{i}\right)\right)} \\
\times\left(\prod_{i=1}^{n}\left(\Lambda_{i}^{(1)}\left(x_{i}\right)-\Lambda_{i}^{(1)}\left(x_{i-1}\right)\right)\left(\Lambda_{i}^{(2)}\left(y_{i}\right)-\Lambda_{i}^{(2)}\left(y_{i-1}\right)\right)\right)^{p-1} 1_{A^{(1)}}(\mathbf{x}) 1_{A^{(2)}}(\mathbf{y})(31)
\end{gathered}
$$

where $C$ is a constant term and $A^{(j)}, j=1,2$ are the sets defined in Proposition 11. We will assume, from now on, that $\mathbf{x} \in A^{(1)}$ and $\mathbf{y} \in A^{(2)}$, as otherwise (30) is trivial. Note that, obviously, if $g_{2} / g_{1}$ is increasing, then

$$
g_{1}(x) g_{2}(y) \leq g_{1}(x \wedge y) g_{2}(x \vee y)
$$

for all $x, y$. Then, assumption (b) ensures that the functions $g_{j}(x)=\lambda_{i}^{(j)}(x)$, $j=1,2$ satisfy (32). In the same way, the functions $g_{j}(x)=e^{-\alpha \Lambda_{i}^{(j)}(x)}, j=1,2$, also satisfy (32) as, in this case,

$$
\frac{e^{-\alpha \Lambda_{i}^{(2)}(x)}}{e^{-\alpha \Lambda_{i}^{(1)}(x)}}=\left(\frac{\bar{F}_{Y_{i}^{(2)}}(x)}{\bar{F}_{Y_{i}^{(1)}}(x)}\right)^{\alpha}
$$

increases with respect to $x$, due to assumption (a). Finally, the functions $g_{j}(x)=e^{-\alpha\left(\Lambda_{i}^{(j)}(x)-\Lambda_{i+1}^{(j)}(x)\right)}$ satisfy the same property too, due to assumption (c). Applying the previous observations to (31), we get:

$$
\begin{aligned}
& \mathbf{f}^{(1)}(\mathbf{x}) \mathbf{f}^{(2)}(\mathbf{y}) \leq C \prod_{i=1}^{n} \lambda_{i}^{(1)}\left(x_{i} \wedge y_{i}\right) \lambda_{i}^{(2)}\left(x_{i} \vee y_{i}\right) \times e^{-\alpha \Lambda_{n}^{(1)}\left(x_{n} \wedge y_{n}\right)} e^{-\alpha \Lambda_{n}^{(2)}\left(x_{n} \vee y_{n}\right)} \\
& \times \prod_{i=1}^{n-1} e^{-\alpha\left(\Lambda_{i}^{(1)}\left(x_{i} \wedge y_{i}\right)-\Lambda_{i-1}^{(1)}\left(x_{i} \wedge y_{i}\right)\right)} e^{-\alpha\left(\Lambda_{i}^{(2)}\left(x_{i} \vee y_{i}\right)-\Lambda_{i-1}^{(2)}\left(x_{i} \vee y_{i}\right)\right)} \\
& \times\left(\prod_{i=1}^{n}\left(\Lambda_{i}^{(1)}\left(x_{i}\right)-\Lambda_{i}^{(1)}\left(x_{i-1}\right)\right)\left(\Lambda_{i}^{(2)}\left(y_{i}\right)-\Lambda_{i}^{(2)}\left(y_{i-1}\right)\right)\right)^{p-1}
\end{aligned}
$$

Let us now set

$$
l_{i}^{(j)}\left(x_{1}, x_{2}\right)=\left(\Lambda_{i}^{(j)}\left(x_{2}\right)-\Lambda_{i}^{(j)}\left(x_{1}\right)\right) 1_{\left\{x_{1}<x_{2}\right\}} .
$$


Based on (33), the claim follows if we prove that

$$
\left.l_{i}^{(1)}\left(x_{1}, x_{2}\right) l_{i}^{(2)}\left(y_{1}, y_{2}\right) \leq l_{i}^{(1)}\left(x_{1} \wedge y_{1}, x_{2} \wedge y_{2}\right) l_{i}^{(2)}\left(x_{1} \vee y_{1}, x_{2} \vee y_{2}\right)\right) .
$$

It is readily seen that $l_{i}^{(1)}\left(x_{1}, x_{2}\right)$ is MTP2, based on the fact that the function $\left(x_{2}-x_{1}\right)_{+}$is MTP2 and that the MTP2 property is preserved under increasing transforms. Then, following similar arguments as in [Shaked and Shanthikumar(2007), p. 291] for the proof of Theorem 6.D.1., it is now sufficient to show that

$$
l_{i}^{(2)}\left(x_{1}, x_{2}\right) l_{i}^{(1)}\left(y_{1}, y_{2}\right) \leq l_{i}^{(2)}\left(y_{1}, y_{2}\right) l_{i}^{(1)}\left(x_{1}, x_{2}\right), \quad\left(x_{1}, x_{2}\right) \leq\left(y_{1}, y_{2}\right) .
$$

Based on assumption (b), this can be done in a similar way as for the proof of Theorem 2(c) in [Badía et al(2018)b, p.180], and it is omitted.

Remark 7 Note that Theorem 16 includes [Belzunce et al(2001), Thm.3.13] as condition (c) holds if its derivative is positive (which is condition (3.11) in [Belzunce et al(2001), Thm.3.13]). Thus, under the same conditions on the trend functions, we extend this theorem to situations where the increment distribution is gamma (which include sequential order statistics, as the increment distribution is exponential in this case). However, more specific results can be obtained in the exponential case and a more detailed analysis will be done in the next section. Our previous theorem also includes the result for trend-renewal processes given in [Badía et al(2018)b, Thm 2(c)], as condition (c) is immediately satisfied if $\Lambda_{i}^{(j)}=\Lambda^{(j)}, \quad i=1, \ldots, n, j=1,2$. It also includes the result for trend-renewal processes given in [Badia et al(2018)b, Thm 3 (c)], in which $\Lambda_{i}^{(j)}=\Lambda^{(j)}, \quad i=1, \ldots, n, j=1,2$ and the increments are gamma distributed with the same shape parameter $p \geq 1$ and (possibly) different scale parameters $\alpha_{1} \geq \alpha_{2} \ldots \geq \alpha_{n}$. To see that, one only needs to use Remark 3 and consider an equivalent representation by taking $\hat{\Lambda}_{i}^{(j)}=\alpha_{i} \Lambda_{i}^{(j)}=\alpha_{i} \Lambda^{(j)}$ (and thus the increment distributions are gamma with scale parameter $=1$ ). If the initial trend vectors $\Lambda^{(\mathbf{j})}, j=1,2$ satisfy the conditions in Theorem 16 , it is readily seen that the vectors $\hat{\boldsymbol{\Lambda}}^{(\mathbf{j})}, j=1,2$ also do, which allows to conclude.

Remark 8 As Theorem 16 is shown for log-concave gamma densities, it could be tempting to think that it could be generalized to any increment distributions having log-concave density. However, the specific conditions for the trend functions in the multivariate likelihood ratio order strongly depend on the specific form of the increment distribution. For instance, if we consider Weibull increments, the conditions given in Theorem 16 are not sufficient to ensure the likelihood ratio comparison results, as we next show. With that aim, let us consider i.i.d. Weibull increments with density:

$$
f(x)=\alpha x^{\alpha-1} e^{-x^{\alpha}}, \quad x \geq 0, \quad \alpha \geq 1 .
$$

If $\Lambda$ is a trend function, then, for $\Lambda_{i}=\Lambda, i=1,2$, the density of $\left(T_{1}, T_{2}\right)$ is given by

$f_{\left(T_{1}, T_{2}\right)}\left(t_{1}, t_{2}\right)=\lambda\left(t_{1}\right) \lambda\left(t_{2}\right) \alpha^{2}\left(\Lambda\left(t_{2}\right)-\Lambda\left(t_{1}\right)\right)^{\alpha-1} \Lambda\left(t_{1}\right)^{\alpha-1} e^{-\left(\Lambda\left(t_{2}\right)-\Lambda\left(t_{1}\right)\right)^{\alpha}} e^{-\Lambda\left(t_{1}\right)^{\alpha}}$. 
Now let us consider $\lambda^{(1)}(x)=2$ and $\lambda^{(2)}(x)=1$ for $x \geq 0$ ( 0 otherwise). Then we have $\Lambda^{(1)}(x)=2 x$ and $\Lambda^{(2)}(x)=x$ and the conditions concerning the trend functions in Theorem 16 are obviously satisfied. Moreover,

$$
\frac{f_{\left(T_{1}^{(2)}, T_{2}^{(2)}\right)}\left(t_{1}, t_{2}\right)}{f_{\left(T_{1}^{(1)}, T_{2}^{(1)}\right)}\left(t_{1}, t_{2}\right)} \propto e^{\left(2^{\alpha}-1\right)\left(\left(t_{2}-t_{1}\right)^{\alpha}+t_{1}^{\alpha}\right)}
$$

It can be easily checked that for $\alpha>1$ and $t_{2}$ fixed, the previous function first decreases and next increases with respect to $t_{1}$.

\section{Multivariate likelihood ratio comparison re- sults for trend-transformed vectors with ex- ponential increments}

As shown in Examples 8 and 9, the case of standard exponential increments (with mean 1) includes Sequential Order Statistics and, in particular, nonhomogeneous pure-birth processes. Multivariate likelihood ratio comparison results were obtained in Belzunce et al. [Belzunce et al(2001)] for the arrival times in a general pure-birth process and in [Zhuang et al(2007)] for sequential order statistics. Such results are particular cases of Theorem 16. Considering standard exponential increments (which are specific gamma ones), we here extend the sufficient conditions from Theorem 16 to more general conditions. When the trend functions share the same support, we also provide necessary and sufficient conditions.

Let us first recall the expression for the density of $\left(T_{1}, \ldots, T_{n}\right)$ given in Proposition 11:

$$
f\left(t_{1}, \ldots, t_{n}\right)=\prod_{i=1}^{n} g_{i}\left(t_{i}\right),-\infty=t_{0}<t_{1}<t_{2}<\cdots<t_{n}, t_{i}<M_{i}
$$

(0 otherwise), where

$$
\begin{aligned}
g_{i}(x): & =\lambda_{i}(x) e^{\Lambda_{i+1}(x)-\Lambda_{i}(x)}, x<M_{i}, i=1, \ldots, n-1 \\
g_{n}(x): & =\lambda_{n}(x) e^{-\Lambda_{n}(x)}, x<M_{n}
\end{aligned}
$$

(0 otherwise).

We now provide the main result of this section.

Theorem 17 Let us consider two trend-transformed independent vectors, $\left(T_{1}^{(j)}, \ldots, T_{n}^{(j)}\right)$, $j=1,2$ with respective trend vectors $\Lambda^{(j)}, j=1,2$, assumed to be absolutely continuous with respective intensity function vectors $\lambda^{(j)}, j=1,2$ and endpoints $\mathbf{M}^{(j)}, j=1,2$. Assume that for each $i=1, \ldots, n$, we have $M_{i}^{(1)} \leq M_{i}^{(2)}$, and that the increments $X_{i}^{(j)}, i=1, \ldots, n, j=1,2$, are identically standard exponentially distributed, that is with common density $f_{i}^{(j)}(x)=e^{-x}, \quad x>0$. For 
each $j=1,2$, let $g_{i}^{(j)}, i=1,2 \ldots, n$ be the functions defined in (38) and (39) corresponding to $\Lambda_{i}^{(j)}, i=1, \ldots, n$. We have the following

(a) $\left(T_{1}^{(1)}, \ldots, T_{n}^{(1)}\right) \leq_{l r}\left(T_{1}^{(2)}, \ldots, T_{n}^{(2)}\right)$ is true under the following condition

$$
g_{i}^{(2)}(x) g_{i}^{(1)}(y) \leq g_{i}^{(2)}(y) g_{i}^{(1)}(x),-\infty<x<y<M_{i}^{(1)}, i=1,2, \ldots, n .
$$

(b) If for each $j=1,2$, the functions $\lambda_{i}^{(j)}, i=1, \ldots, n$ share the same support, then $\left(T_{1}^{1}, \ldots, T_{n}^{1}\right) \leq_{l r}\left(T_{1}^{2}, \ldots, T_{n}^{2}\right)$ is equivalent to $(40)$.

Proof. Let $\mathbf{f}^{(j)}$ be the density function of $\left(T_{1}^{(j)}, \ldots, T_{n}^{(j)}\right)$ for $j=1,2$. For the likelihood ratio order to hold, we need to check that

$$
\mathbf{f}^{(1)}(\mathbf{x}) \mathbf{f}^{(2)}(\mathbf{y}) \leq \mathbf{f}^{(1)}(\mathbf{x} \wedge \mathbf{y}) \mathbf{f}^{(2)}(\mathbf{x} \vee \mathbf{y}), \quad \text { for all } \mathbf{x} \text { and } \mathbf{y} \text {. }
$$

Assuming (40) to be true, we always have $g_{i}^{(1)}\left(x_{i}\right) g_{i}^{(2)}\left(y_{i}\right) \leq$ $g_{i}^{(1)}\left(x_{i} \wedge y_{i}\right) g_{i}^{(2)}\left(x_{i} \vee y_{i}\right)$ when taking arbitrary $x_{i}$ and $y_{i}$. This entails (41) based on (37), and point (a) is true. For point (b), assume (41) to hold and let us show (40). Assume also the following.

There exists $i=1, \ldots, n$ and $0<x<y$ such that $g_{i}^{(2)}(x) g_{i}^{(1)}(y)>0$.

(as if there is no such $i$ and $(x, y)$, the result is clear). Let $(i, x, y)$ fulfills $(42)$. Note that this means that $\lambda_{i}^{(1)}(y) \lambda_{i}^{(2)}(x)>0$ and hence $\lambda_{1}^{(1)}(y) \lambda_{1}^{(2)}(x)>0$, as for each $j=1,2$, the functions $\lambda_{i}^{(j)}, i=1, \ldots, n$ share the same support. Moreover, based on the preservation of the likelihood ratio order under marginalization, (41) implies that $T_{1}^{(1)} \leq_{l r} T_{1}^{(2)}$. As the density of $T_{1}^{(j)}$ is $g_{1}^{(j)}(x)=\lambda_{1}^{(j)}(x) e^{-\Lambda_{1}^{(j)}(x)}, x \in \mathbb{R}$, we now get

$$
0<\lambda_{1}^{(1)}(y) \lambda_{1}^{(2)}(x) e^{-\Lambda_{1}^{(1)}(y)} e^{-\Lambda_{1}^{(2)}(x)} \leq \lambda_{1}^{(1)}(x) \lambda_{1}^{(2)}(y) e^{-\Lambda_{1}^{(1)}(x)} e^{-\Lambda_{1}^{(2)}(y)} .
$$

From the previous inequality and the fact that for each $j$, the functions $\lambda_{i}^{(j)}$, $i=1, \ldots, n$ share the same support, we now derive the following.

$$
\begin{aligned}
& \text { If }(i, x, y) \text { fulfills }(42) \text {, then } \lambda_{i}^{(j)}(x)>0 \text { and } \lambda_{i}^{(j)}(y)>0 \\
& \text { for all } i=1, \ldots, n \text { and } j=1,2 \text {. }
\end{aligned}
$$

Now consider $m^{(j)}:=\inf \left\{x \in \mathbb{R} \mid \Lambda_{i}^{(j)}(x)>0\right\}, j=1,2$. If $m^{(j)}>-\infty$, we can assume, without lost of generality that $\lambda_{i}^{(j)}(x)=0, x \leq m^{(j)}$ (this only entails to modify the definition of the $\lambda_{i}^{(j)}$ on a set with Lebesgue measure 0 , if needed). By (42), we know that $x \in\left(m^{(2)}, M^{(2)}\right)$ and $y \in\left(m^{(1)}, M^{(1)}\right)$. By definition of $m^{(2)}$, we now have $\Lambda_{1}^{(2)}(x)=\int_{m^{(2)}}^{x} \lambda^{(2)}(y) d y>0$, which entails that $\lambda_{1}^{(2)}$ does not almost surely cancel on $\left(m^{(2)}, x\right)$. It is hence possible to construct $x_{k}, k=1, \ldots, i-1$ such that $m^{(2)}<x_{1}<\ldots<x_{i-1}<x$ with $\lambda_{1}^{(2)}\left(x_{k}\right)>0$. 
In the same way, we can construct $y_{k^{\prime}}, k^{\prime}=i+1, \ldots, n$ such that $y<y_{i+1}<$ $\ldots<y_{n}<M^{(1)}$ with $\lambda_{1}^{(1)}\left(y_{k^{\prime}}\right)>0$. Based on (43) and $x_{k}<y_{k^{\prime}}$ (because $x<y)$, we derive that $\lambda_{i}^{(j)}\left(x_{k}\right)>0$ and $\lambda_{i}^{(j)}\left(y_{k^{\prime}}\right)>0$ for all $k, k^{\prime}, i$ and $j$. Taking $\mathbf{x}:=\left(x_{1}, \ldots, x_{i-1}, y, y_{i+1}, \ldots, y_{n}\right)$ and $\mathbf{y}:=\left(x_{1}, \ldots, x_{i-1}, x, y_{i+1}, \ldots, y_{n}\right)$ in (41), we derive from (37) that $g_{i}^{(1)}(y) g_{i}^{(2)}(x) \leq g_{i}^{(2)}(x) g_{i}^{(1)}(y)$, as asserted.

Remark 9 Based on Example 8, the previous result gives necessary conditions for likelihood ratio comparisons of sequential order statistics (among others), which become necessary under mild conditions. Using the notations of Example 8 , let $\Lambda_{i}^{\star}$ and $\Lambda_{i}, i=1, \ldots, n$ stand for the cumulative hazard rate functions corresponding to $G_{i}^{\star}$ and $G_{i}$, respectively. Then, it is easy to check that

$$
\Lambda_{i}(x)=(n-i+1) \Lambda_{i}^{\star}(x), \quad i=1, \ldots, n,
$$

due to (1) and (15). Applying Theorem 17 in this setting allows us to recover Theorem 3.1 from [Zhuang et al(2007)], and Theorem 17 hence enlarges this result.

When considering non-homogeneous pure birth processes, we are able to find situations not included in the general results provided in [Belzunce et al(2001)], as the following example shows.

Example 18 Let $\left(T_{1}^{(j)}, T_{2}^{(j)}\right), j=1,2$, be two trend-transformed independent vectors with corresponding intensity functions $\lambda_{1}^{(1)}(x)=2+x, \lambda_{2}^{(1)}(x)=1+x / 2$, $\lambda_{1}^{(2)}=1$ and $\lambda_{2}^{(2)}=1 / 2, x>0$, and both with standard exponential increments. Let us consider $g_{i}^{(j)}$, as defined in (38) and (39). A straightforward calculus shows us that

$$
\frac{g_{1}^{(2)}(x)}{g_{1}^{(1)}(x)}=\frac{g_{2}^{(2)}(x)}{g_{2}^{(1)}(x)}=\frac{1}{2+x} e^{x / 2+x^{2} / 4}, \quad x>0
$$

which is an increasing function on $(0, \infty)$. From Theorem (17), we thus have that $\left(T_{1}^{(1)}, T_{2}^{(1)}\right) \leq_{l r}\left(T_{1}^{(2)}, T_{2}^{(2)}\right)$. However, we cannot use the result for nonhomogeneous pure-birth processes given in [Belzunce et al(2001), Thm.3.13], as $\frac{\lambda_{1}^{(2)}(x)}{\lambda_{1}^{(1)}(x)}=\frac{1}{2+x}$ is a decreasing function.

As an illustration of Theorem 17, let us consider a given intensity function $\lambda$ and its corresponding $\Lambda$. We now particularize the previous results to trend functions of the form

$$
\Lambda_{i}=a_{i} \Lambda, \quad a_{i}>0, i=1, \ldots, n,
$$

thus including generalized order statistics and extended Pólya processes, as explained at the end of Example 8.

In the specific case where the two trend-transformed vectors share the same $\Lambda$ in (44), but the coefficients $a_{i}^{(j)}, j=1,2$ differ, the previous proposition 
allows to easily write down necessary and sufficient conditions in terms of the coefficients to get the likelihood ratio comparison results. The reasoning is clear and the proof is omitted.

Corollary 19 For a given intensity function $\lambda$, let us consider two trendtransformed independent vectors, $\left(T_{1}^{(j)}, \ldots, T_{n}^{(j)}\right), j=1,2$ with respective trend functions $a_{i}^{(j)} \Lambda, i=1, \ldots, n, j=1,2$ and both with standard exponential increments. Then $\left(T_{1}^{(1)}, \ldots, T_{n}^{(1)}\right) \leq_{l r}\left(T_{1}^{(2)}, \ldots, T_{n}^{(2)}\right)$ if and only if the following inequalities hold:

$$
\begin{aligned}
a_{n}^{(1)} & \geq a_{n}^{(2)} \\
a_{i+1}^{(1)}-a_{i}^{(1)} & \leq a_{i+1}^{(2)}-a_{i}^{(2)}, i=1,2, \ldots, n-1
\end{aligned}
$$

Remark 10 This set of conditions is similar to the sufficient conditions appearing in [Belzunce et al(2001), Thm 3.13] for theses specific pure-birth processes. Notice that we show that they are also necessary. However, they are quite restrictive. Indeed, we can rewrite these inequalities as

$$
0 \leq a_{n}^{(1)}-a_{n}^{(2)} \leq a_{n-1}^{(1)}-a_{n-1}^{(2)} \leq \ldots \leq a_{1}^{(1)}-a_{1}^{(2)} .
$$

If we consider EPPs with the supplementary assumption $a_{1}^{(1)}=a_{1}^{(2)}$, this condition entails that $a_{i}^{(1)}=a_{i}^{(2)}$ for all $i \in\{1, \ldots, n\}$.

We now derive necessary and sufficient conditions for comparing specific Extended Pólya processes in the likelihood ratio ordering, please see Example 5 for the notations. The first result is for generalized Pólya processes. It is a direct consequence of Corollary 19 and it is stated without proof.

Corollary 20 Let us consider two generalized Pólya processes $\left(h^{(j)}, \lambda\right), j=$ 1,2 , with $h^{(j)}(i)=\alpha_{j} i+\beta_{j}, i=1,2, \ldots, \alpha_{j} \geq 0, \beta_{j}>0, j=1,2$. Then we have $\left(T_{1}^{(1)}, \ldots, T_{n}^{(1)}\right) \leq_{l r}\left(T_{1}^{(2)}, \ldots, T_{n}^{(2)}\right)$ for some $n \geq 2$ if and only if $\alpha_{1} \leq \alpha_{2}$ and $\beta_{1}-\beta_{2} \geq\left(\alpha_{2}-\alpha_{1}\right)(n-1)$.

Remark 11 From the previous result, we deduce easily that the likelihood ratio ordering between two generalized Pólya processes holds for all $n$ if and only if $\alpha_{1}=\alpha_{2}$ and $\beta_{1} \geq \beta_{2}$.

In the following result, we derive stochastic comparisons for extended Pólya processes sharing the same $\lambda$ with non-linear functions $h(i)=q^{i}+\beta$.

Corollary 21 Let us consider two extended Pólya processes $\left(h^{(j)}, \lambda\right), j=1,2$, with $h^{(j)}(n)=q_{j}^{n}+\beta_{j}$, where $q_{j}>0, \beta_{j} \geq 0$. Let $\left(T_{n}^{(j)}\right)_{n=1,2, \ldots}, j=1,2$ be their respective arrival times. We have that $\left(T_{1}^{(1)}, \ldots, T_{n}^{(1)}\right) \leq_{\operatorname{lr}}\left(T_{1}^{(2)}, \ldots, T_{n}^{(2)}\right)$ for some $n \geq 2$ if and only if $q_{1} \leq q_{2}$ and $q_{1}^{n-1}+\beta_{1} \geq q_{2}^{n-1}+\beta_{2}$, with the additional condition $q_{2}^{n-1}-q_{1}^{n-1} \geq q_{2}^{n-2}-q_{1}^{n-2}$ if $q_{2}<1$. 
Proof. We apply Corollary 19 with $a_{i}^{(j)}=h^{(j)}(i-1), i=1, \ldots, n$. Condition $q_{1}^{n-1}+\beta_{1} \geq q_{2}^{n-1}+\beta_{2}$ is just equivalent to (45). The point hence is to show that (46) is equivalent to the other conditions, that is to $q_{1} \leq q_{2}$ with the additional condition $q_{2}^{n-1}-q_{1}^{n-1} \geq q_{2}^{n-2}-q_{1}^{n-2}$ if $q_{2}<1$. Now (46) is just equivalent to

$$
q_{1}^{i}-q_{2}^{i}-q_{1}^{i-1}+q_{2}^{i-1} \leq 0
$$

for all $1 \leq i \leq n-1$. The previous condition for $i=1$ gives us $q_{1} \leq q_{2}$. Thus, this is a necessary condition. On the other hand, if we divide the previous expression by $q_{1}^{i-1}$ and rearrange terms, we have the following equivalent inequality:

$$
\left(1-q_{2}\right)\left(\frac{q_{2}}{q_{1}}\right)^{i-1} \leq 1-q_{1}
$$

If $q_{2} \geq 1$, the expression on the left-hand side is decreasing with respect to $i$ and hence maximal for $i=1$. Thus $q_{1} \leq q_{2}$ is a sufficient condition to derive (47). If $q_{2}<1$, the previous expression is increasing on $i$, thus to have (47) for all $i$, we need to check it for $i=n-1$, which gives the additional condition for $q_{2}<1$.

Remark 12 Observe that, considering $q_{2}=1$, the previous likelihood ratio comparison result is valid for all $n$ if $q_{1} \leq 1$ and $\beta_{1}-\beta_{2} \geq 1$. As a consequence, by taking $\beta_{2}=\beta_{1}-1$ the arrival times in an $\operatorname{EPP}\left(h^{(1)}, \lambda\right)$ with $h^{(1)}(n)=q_{1}^{n}+\beta_{1}$, $q_{1}<1, \beta_{1}>0$ are bounded (in the multidimensional likelihood ratio sense) by the ones of a non-homogeneous Poisson process with intensity $\left(1+\beta_{2}\right) \lambda=\beta_{1} \lambda$. On the other hand, for $q_{2} \neq 1$, the previous result cannot be valid for all $n$, unless $q_{2}=q_{1}$ and $\beta_{1} \geq \beta_{2}$. Indeed, if $q_{1}<q_{2}<1$, (48) cannot hold for all $i$,

and if $q_{1}<q_{2}$ and $q_{2}>1$ the condition $q_{1}^{n-1}+\beta_{1} \geq q_{2}^{n-1}+\beta_{2}$ cannot hold for all $n$, as we can rewrite this as

$$
q_{2}^{n-1}\left(1-\left(\frac{q_{1}}{q_{2}}\right)^{n-1}\right) \leq \beta_{1}-\beta_{2}
$$

\section{Concluding remarks and perspective}

In this paper, we have proposed a new model which includes many others from the literature, and hence allows to treat in a unified way. Multivariate stochastic comparison results have also been provided in the usual and likelihood ratio orders, which extend others, previously developed under specific assumptions. Clearly, there remain many other points to study for trend-transformed independent vectors. First, we could consider multivariate stochastic comparison with respect to other stochastic orders such as the multivariate hazard rate order or the dispersive and mean residual life orders, trying to extend the results from [Belzunce et al(2001), Belzunce et al(2005)] on these points. Also, Proposition 12 could be extended to study other dependence properties between the epoch or inter-epoch times. 
It might be of interest too to study the counting process $\left(N_{t}\right)_{t \geq 0}$ corresponding to a trend-transformed independent sequence, that is such that the vector of the $n$ first arrival times is a trend-transformed independent vector. As for the distribution of the number of arrivals $N_{t}$ in a fixed time interval $[0, t]$, it does not seem to be available in a nice full form expression in a general setting. Maybe one could look at specific parameterized cases? Beyond that, it should however be possible to extend the stochastic comparisons results for the arrival times in trend-transformed independent vectors ( $n$ fixed) to the arrival times in two counting processes associated to two different trend-transformed independent sequences, as is done in [Badía et al(2018)b, Thm. 3 (e),(f)] for two different trend-renewal processes. The practical applications of those results would be relevant for comparing cumulative shock models associated to those counting processes, as is done in [Badía et al(2018)b, Application 4.1] for shock processes associated to trend-renewal processes.

Finally, for a practical use of the new model in an application context, there is a clear need to develop estimation procedures. As noted by one referee, based on the fact that a trend-transformed independent vector implies $n$ trend functions and $n$ distribution functions in its definition, it is essential to consider parameterizing these functions. Considering Remark 3, we already know that we can impose that the $F_{i}$ 's should have expected values equal to 1 , as in [Lindqvist(2003)] for trend-renewal processes. Now, to quote only one possible parametrization among many others, one could consider gamma distributions $\mathcal{G}\left(a_{i}, a_{i}\right)$ for the $F_{i}$ 's with $\mathbb{E}\left(X_{i}\right)=1$ and $\operatorname{var}\left(X_{i}\right)=1 / a_{i}$. Parameter $a_{i}$ could next be parameterized (example: $\alpha_{i}=\sigma_{1} \sigma_{2}^{i}$ ). As for the trend functions, we could envision the classical power-law shape function $\Lambda_{i}(t)=\alpha_{i} t^{\beta_{i}}$. Next, according to the context, one parameter among $\alpha_{i}$ and $\beta_{i}$ could be considered as fixed (say $\beta_{i}$ for instance) and the other one to have a parametric shape (example : $\alpha_{i}=\gamma_{1} i+\gamma_{2}$ ). Then, one could try to estimate the parameters $\left(\sigma_{1}, \sigma_{2}, \beta, \gamma_{1}, \gamma_{2}\right)$ through maximizing the likelihood function, which is easy to write down from the joint density function given in (11).

Clearly these estimation procedures deserve further work, firstly to understand which kind of parametrization to be used according to the data (and for this, experts advice might be of great interest), secondly to explore the maximum likelihood method (or others) in this context.

\section{Acknowledgments}

The authors would like to thank the anonymous referees for their helpful comments. This work has been supported by the Spanish research projects MTM201563978 and PGC2018-094964-B-100 (MINECO/FEDER). The first and third authors acknowledge the support of DGA S41-17R and E26-17R, respectively. 


\section{References}

[Asfaw and Lindqvist(2015)] Asfaw ZG, Lindqvist BH (2015) Extending minimal repair models for repairable systems: A comparison of dynamic and heterogeneous extensions of a nonhomogeneous Poisson process. Reliability Engineering 83 System Safety 140:53 - 58

[Badía et al(2018a)] Badía F, Mercier S, Sangüesa C (2018) Extensions of the Generalized Pólya Process. Methodology and Computing in Applied Probability. Available online.

[Badía et al(2018)b] Badía F, Sangüesa C, Cha J (2018) Stochastic comparisons and multivariate dependence for the epoch times of trend renewal processes. Journal of Multivariate Analysis 168: 233-253.

[Badía et al(2018c)] Badía F, Sangüesa C, Cha J (2018) Stochastic comparisons and ageing properties of generalized Pólya processes. Journal of Applied Probability 55(1): 174-184.

[Berman (1981)] Berman M (1981) Inhomogeneous and modulated gamma processes. Biometrika 68(1): 143-152.

[Beutner and Kamps(2008)] Beutner E, Kamps U (2008). Models of ordered data and products of beta random variables. Advances in Mathematical Statistical Modeling. Eds Mínguez R, Sarabia JM, Balakrishnan N, Arnold B, 101-106.

[Belzunce et al(2001)] Belzunce F, Lillo RE, Ruiz JM, Shaked M (2001) Stochastic comparisons of nonhomogeneous processes. Probability in the Engineering and Informational Sciences 15: 199-224.

[Belzunce et al(2005)] Belzunce F, Mercader J, Ruiz J (2005) Stochastic comparisons of generalized order statistics. Probability in the Engineering and Informational Sciences 19: 99-120.

[Boyer and $\operatorname{Roux}(2016)]$ Boyer M, Roux P (2016) A common framework embedding network calculus and event stream theory, https://hal.archivesouvertes.fr/hal-01311502, working paper or preprint.

[Cha(2014)] Cha JH (2014) Characterization of the generalized Pólya process and its applications. Advances in Applied Probability 46: 1148-1171.

[Cha and Finkelstein(2017)] Cha JH, Finkelstein M (2017) New shock models based on the generalized Pólya process. European Journal of Operations Research 251: 1148-1171.

[Cramer and Kamps (2003)] Cramer E, Kamps U (2003) Marginal distributions of sequential and generalized order statistics. Metrika 58: 293-310.

[Embrechts and Hofert(2013)] Embrechts P, Hofert M (2013) A note on generalized inverses. Mathematical Methods of Operations Research 77(3):423-432. 
[Kamps(1995a)] Kamps U (1995) A concept of generalized order statistics. Journal of Statistical Planning and Inference 48(11):1-23.

[Kamps(1995b)] Kamps U (1995) A concept of generalized order statistics. Teubner Skripten zur Mathematischen Stochastik. [Teubner Texts on Mathematical Stochastics], Teubner BG, Stuttgart.

[Karlin and Rinott(1980)] Karlin, S. and Rinott, J.(1980) Classes of ordering measures and related correlation inequalities I. Multivariate totally positive distributions. Journal of Multivariate Analysis 10: 467-498.

[Lenz(2008)] Lenz B (2008) Markovian simple counting processes and models of ordered random variables. Dissertation, Fakultät für Mathematik, Informatik und Naturwissenschaften der Rheinisch-Westfälischen, Technischen Hochschule, Aachen.

[Lindqvist(2003)] Lindqvist BH, Elvebakk G and Heggland K (2003) The trendrenewal process for statistical analysis of repairable systems. Technometrics 45: 31-44.

[Marshall and Olkin (2007)] Marshall, A.W. and Olkin, I. (2007). Life Distributions. Springer, New York.

[Müller and Stoyan(2002)] Müller A, Stoyan D (2002) Comparison methods for stochastic models and risks. Wiley Series in Probability and Statistics, Wiley, Chichester.

[Papadatos(1995)] Papadatos N (1995) Intermediate order statistics with applications to nonparametric estimation. Statistics 83 Probability Letters 22: 231-238.

[Shaked and Shanthikumar(2007)] Shaked M, Shanthikumar JG (2007) Stochastic orders. Springer Series in Statistics, Springer, New York.

[Torrado(2012)] Torrado N, Lillo, RE, Wiper, MP (2012) Sequential Order Statistics: Ageing and Stochastic Orderings. Methodology and Computing in Applied Probability 14: 579-596.

[Zhuang et al(2007)] Zhuang, W. Hu, T (2007). Multivariate stochastic comparisons of sequential order statistics. Probability in the Engineering and Informational Sciences 21(1): 47-66. 